

\title{
Hardening/softening behaviour in non-linear oscillations of structural systems using non-linear normal modes
}

\author{
Cyril Touzé, Olivier Thomas, Antoine Chaigne
}

\section{To cite this version:}

Cyril Touzé, Olivier Thomas, Antoine Chaigne. Hardening/softening behaviour in non-linear oscillations of structural systems using non-linear normal modes. Journal of Sound and Vibration, 2004, 273 (1-2), pp.77-101. 10.1016/j.jsv.2003.04.005 . hal-00830693

\section{HAL Id: hal-00830693}

\section{https://hal-ensta-paris.archives-ouvertes.fr/hal-00830693}

Submitted on 5 Oct 2015

HAL is a multi-disciplinary open access archive for the deposit and dissemination of scientific research documents, whether they are published or not. The documents may come from teaching and research institutions in France or abroad, or from public or private research centers.
L'archive ouverte pluridisciplinaire HAL, est destinée au dépôt et à la diffusion de documents scientifiques de niveau recherche, publiés ou non, émanant des établissements d'enseignement et de recherche français ou étrangers, des laboratoires publics ou privés. 


\title{
Hardening/softening behaviour in non-linear oscillations of structural systems using non-linear normal modes
}

\author{
C. Touzé*, O. Thomas, A. Chaigne \\ ENSTA - UME, Chemin de la Hunière, 91761 Palaiseau Cedex, France
}

\begin{abstract}
The definition of a non-linear normal mode (NNM) as an invariant manifold in phase space is used. In conservative cases, it is shown that normal form theory allows one to compute all NNMs, as well as the attendant dynamics onto the manifolds, in a single operation. Then, a single-mode motion is studied. The aim of the present work is to show that too severe trunc ature using a single linear mode $\mathrm{c}$ an lead to erroneous results. Using single-non-linear mode motion predic ts the correc tbehaviour. Hence, the nonlinear change of co-ordinates allowing one to pass from the linear modal variables to the normal ones, linked to the NNMs, defines a framework to properly truncate non-linear vibration PDEs. Two examples are studied: a disc rete system (a mass connec ted to two springs) and a continuous one (a linear EulerBernoulli beam resting on a non-linear elasticfoundation). For the latter, a comparison is given between the developed method and previously published results.
\end{abstract}

\section{Introduction}

One main characteristic in non-linear oscillations is the frequency dependence on vibration amplitude, which can be of hardening or softening type [1]. As a system vibrates with large amplitudes, it is a well-established fact that the mode shapes also depend on vibration amplitude (see e.g. Ref. [2]). Hence, a correct modelling has to tackle these two difficulties. Moreover, drastic reductions of the dimension of the studied phase space is a goal that is often pursued. Indeed, deriving accurate reduced order models is an appreciated methodology since it allows one to predict carefully a number of different dynamical features through simple calculations. It gives an 
alternative to crude numerical computations, as well as insight into and fine comprehension of the model studied.

When considering a single-mode motion, a common - though crude - approximation, consists of projecting the non-linear partial differential equations (PDEs) governing the motion onto the considered linear eigenmode. Unfortunately, this method sometimes gives wrong qualitative results about the trend of non-linearity. More precisely, it has been frequently observed that this truncature predicts a hardening behaviour whereas experimental measures display a softening one. This problem has been encountered in circular cylindrical shells vibrations for a long time. A complete and interesting review of the literature on this problem, as well as experimental measures can be found in Refs. [3,4]. The same problematic was also met in the non-linear vibrations of buckled beams [5]. As it will be shown next, this is specific to quadratic non-linearity.

In a series of papers, Nayfeh et al. pointed out that these too severe truncatures (referred to as "the discretization method" in their papers) can lead to erroneous quantitative as well as qualitative results [6-9]. Methodologies were proposed in order to overcome these errors, by using a perturbation technique (the method of multiple scales) directly into the non-linear PDE [5,8], or by considering a spatial adjustable function in addition to the linear eigenmode [9].

An alternative, but in fine equivalent approach to these difficulties, is proposed by Shaw and Pierre [10,11], by constructing a mathematical framework in order to derive the definition of a non-linear normal mode (NNM), a concept that was proposed by Rosenberg [12] and used by several different authors, see for example Refs. [13-19]. A NNM is defined as an invariant manifold in phase space, which is tangent to its linear counterpart (the linear eigenspace) at the origin. Shaw and Pierre used centre manifold reduction theorem $[10,11,19]$ in order to compute the geometry of these invariant manifolds. The main idea outlined is that, at the non-linear stage, one has to span the phase space with invariant manifolds rather than still using linear eigenspaces. This leads to definition of a single-mode motion with an amplitude-dependent mode shape.

The objective of this contribution is to show the equivalence of these methods, which found their theoretical foundations in Poincaré and Poincaré-Dulac's theorems [20-23]. Although these results are not new, a systematic procedure is proposed, allowing one to derive reduced order models that capture the effective trend of non-linearity without increasing the complexity, and that display amplitude-dependent mode shapes. Normal form theory defines new co-ordinates linked to the invariant manifolds, as well as the non-linear relation between these new co-ordinates and the initial (modal) ones. It results in a curved invariant-based span of the phase space. Moreover, as a result of this operation, the attendant dynamics onto the manifolds is simply given by the normal form of the problem at hand.

Normal form theory has been widely used in the past few years. To the authors' knowledge, it has first been introduced to solve non-linear vibration problems by Jezequel and Lamarque $[14,15]$. However, it has always been derived under its complex-diagonal form, as recommended by the mathematics. The book by Nayfeh [24] sets down normal form theory as a perturbation method amongst others and yields comparison with the multiple-scales method. Pellicano and Mastroddi used it in Ref. [25] to specify the idea of non-linear superposition, which was incorrectly proposed in Ref. [10]. Morino et al. [26] called it a "Lie transform", Amabili et al. [3] derived it in a general manner for shell vibrations problems. All these calculations used complexdiagonal form. Here, real blocks are used at the linear stage. This allows one to derive all the 
calculations in a framework that is more directly readable for the mechanics community, as well as to directly compare the results with the method developed in Refs. [10,11,16,27,28].

The calculations presented are general and derived for a set of $N$ non-linear oscillators, with $N$ as large as required. This is to stress that the method of real normal form, as formulated here, should be systematically used in the procedure for studying non-linear vibration problems. More precisely, it is suggested to:

(i) Perform the discretization of the system through projection onto the complete linear mode basis.

(ii) Compute the non-linear change of co-ordinates, allowing one to pass from linear modal co-ordinates to normal co-ordinates, linked to the NNMs.

It is only when these two steps have been achieved that one could state the problem of an efficient truncature.

Simple results will be shown here for single non-linear modal motions, as a first application of this general procedure. It is shown that taking the NNMs into account can change quantitatively, as well as qualitatively, the hardening or softening behaviour of the considered mode. These theoretical results are illustrated by two examples. First, a discrete system is studied. Secondly, a linear Euler-Bernoulli beam resting on a non-linear elastic foundation is considered. This simple example has already been tackled by many authors (see e.g. Refs. [5,8,9,29-31]). A detailed comparison is provided here between the method of invariant manifold and the one proposed in Refs. [5,8], which will allow illustration of their equivalence.

\section{Theory}

\subsection{Framework}

Non-linear oscillations of structural undamped systems with geometric quadratic and cubic non-linearities are considered in the following. The motion of continuous systems is assumed to be governed by partial differential equations (e.g. Von Kármán type equations for beams, plates, archs and shells), subjected to boundary conditions. Because of the orthogonality properties of the linear eigenmodes, it is a common method to separate time and space variables, and to project the PDE onto the linear modes basis. Letting

$$
w(\mathbf{x}, t)=\sum_{p=1}^{+\infty} X_{p}(t) \Phi_{p}(\mathbf{x}),
$$

where $w$ is the displacement, $\mathbf{x}$ the spatial variables, $X_{p}$ the modal co-ordinate associated to the linear eigenmode $\Phi_{p}$, the problem is equivalent to

$$
\forall p=1, \ldots,+\infty: \ddot{X}_{p}+\omega_{p}^{2} X_{p}+\sum_{i=1}^{+\infty} \sum_{j \geqslant i}^{+\infty} g_{i j}^{p} X_{i} X_{j}+\sum_{i=1}^{+\infty} \sum_{j \geqslant i}^{+\infty} \sum_{k \geqslant j}^{+\infty} h_{i j k}^{p} X_{i} X_{j} X_{k}=0 .
$$

The coefficients $g_{i j}^{p}$ and $h_{i j k}^{p}$ arise from the projection of the non-linear terms of the PDE onto the linear modes. Damping is omitted, mainly because only lightly damped systems are considered, 
for which $\omega_{p} \gg \mu_{p}$ (where $\mu_{p}$ stands for the modal damping). A finite number $N$ of linear modes is then retained in the analysis, where $N$ is assumed to be large.

The formulation given in Eqs. (1) and (2) has been retained with the purpose of application to continuous structures. However, discrete systems can also be treated. The assumption of undamped system is central to this work since the eigenspectrum of the PDE enforced the structure of Eq. (2), as well as its normal form [21-23]. In particular, gyroscopic systems, that are conservative, can be treated with the proposed method: one has just to express the temporal problem into the form of Eq. (2), which is possible for gyroscopic systems as long as they are stable, since their eigenspectrum is composed of purely imaginary complex conjugates.

The idea of defining a non-linear normal mode is to extend the decoupling of the linear eigenspaces exhibited at the linear stage. Letting $g_{i j}^{p} \equiv h_{i j k}^{p} \equiv 0$ in Eq. (2), and initiating a motion along the $p$ th eigendirection results in a motion which is always contained within it. This is the invariance property one would be able to extend to the non-linear regime.

Moreover, a physical definition of a NNM, as it was given for example by Rosenberg [12], is a motion of the structure such that all points pass through their static-equilibrium positions and achieve their maximum displacements at the same time. Thus, a physical NNM motion can be mathematically described by a single displacement-velocity pair governed by a single oscillator. In phase space, such motions occur on two-dimensional invariant manifolds only. The idea is then to span the phase space with the invariant manifolds, and no longer with the linear eigenspaces.

Normal form theory has been developed for a long time and relies upon ideas that are summarized in Poincaré and Poincaré-Dulac's theorems (see Refs. [21-23] and references therein). However, in vibration theory, it has generally been used as a perturbation method amongst others [24]. Here it will be used as a general tool that enables spanning the phase space with invariant manifolds, hence generalizing the approach used in the definition of the NNMs by other authors $[10,11,14]$. To specify the idea before entering the calculations, the situation is sketched in Fig. 1, where the phase space in the vicinity of the origin (which is the position of the structure at rest) is represented. Linear normal modes are two-dimensional orthogonal planes: they are represented here by lines for convenience, and only two of them are shown, although the number of modes is equal to $N$. When the system vibrates with large amplitude and enters the non-linear regime, the linear eigenspaces are not invariant anymore. Non-linear normal modes are invariant manifolds that are tangent to the linear eigenmodes at the origin. The first two modes: $\mathscr{M}_{1}$ and $\mathscr{M}_{2}$ are represented. Computing the real normal form allows one to

- Express the non-linear relationship between modal co-ordinates $\left(X_{p}, Y_{p}\right)$ (where $Y_{p}=\dot{X}_{p}$ is the velocity) and the new-defined normal co-ordinates $\left(R_{p}, S_{p}\right)$. These new co-ordinates are linked to the invariant manifolds, and thus the dynamics is directly expressed in a curved grid, also represented.

- Get the expression of the dynamics into the curved grid, with the normal co-ordinates.

The main results of these calculations are summarized in the next section. The complete demonstration is not given for the sake of brevity. The interested reader can find it in [32]. The case of two degree-of-freedom (d.o.f.) systems has already been treated with this formality in Ref. [33], and simple continuous structures (beams and plates) in Ref. [34]. 


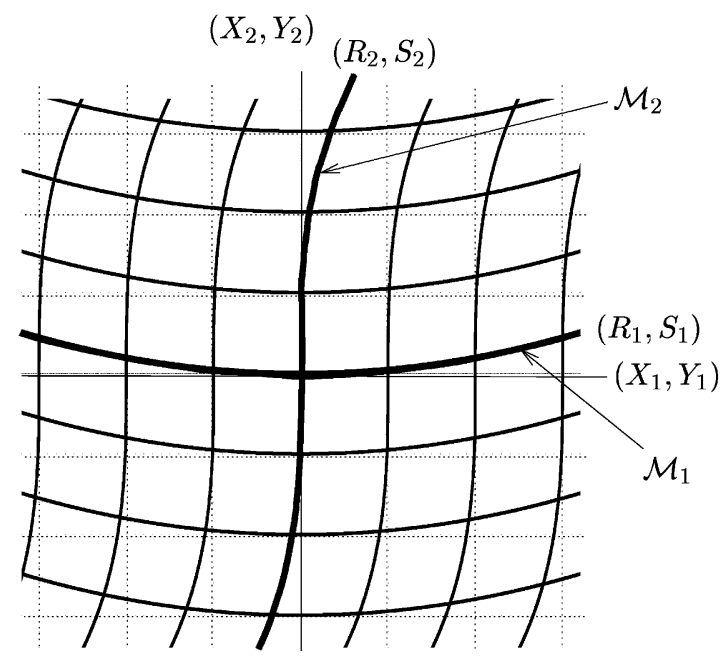

Fig. 1. Schematical representation of the phase space near the position of the system at rest, located at the origin. Orthogonal lines represent the first two linear eigenmodes, graduated by the modal co-ordinates $\left(X_{p}, Y_{p}\right)$. The curved heavy lines, which are tangential to the axes at the origin, are the invariant manifolds (the NNMs), graduated by the normal co-ordinates $\left(R_{p}, S_{p}\right)$. Normal form theory allows one to define the dynamics into the curved grid, in the phase space spanned by the NNMs.

\subsection{Main results}

Computing the normal form of a dynamical system consists of exhibiting a non-linear change of co-ordinates which cancels non-resonant terms [20,21]. Here, a third order asymptotic development is expressed. In this section, it is assumed that no internal resonance is present in the eigenspectrum $\left\{ \pm \mathrm{i} \omega_{p}\right\}$. The question of internal resonance is addressed in Section 2.3. The calculations, detailed in Ref. [32], are performed with the following general rules. First, Eq. (2) is set into its first order form by using the velocity $Y_{p}=\dot{X}_{p}$ as second variable. Then, at linear stage, the system is not diagonalized to keep real expressions. Oscillator-blocks, of the form

$$
\left.\begin{array}{cc}
0 & 1 \\
-\omega_{p}^{2} & 0
\end{array}\right)
$$

are kept instead of diagonal, complex representation. This is the only difference with the approach used by Jezequel and Lamarque [14,15] and other authors Refs. [3,24-26]. It is however important since it allows one to keep the real oscillator form throughout the calculations: dynamical equations will always begin with $\ddot{X}+\omega^{2} X+\cdots$, which is more readable for the mechanicians community. As a consequence, velocity-dependent terms arise in the expressions. The near-identity change of co-ordinates is sought by expressing its more general form, involving all the state-space variables (and thus including 
the velocities), and then computing the correspondant coefficients [32]. It reads, up to order three: $\forall p=1, \ldots, N$ :

$$
\begin{aligned}
X_{p}= & R_{p}+\sum_{i=1}^{N} \sum_{j \geqslant i}^{N}\left(a_{i j}^{p} R_{i} R_{j}+b_{i j}^{p} S_{i} S_{j}\right) \\
& +\sum_{i=1}^{N} \sum_{j \geqslant i}^{N} \sum_{k \geqslant j}^{N} r_{i j k}^{p} R_{i} R_{j} R_{k}+\sum_{i=1}^{N} \sum_{j=1}^{N} \sum_{k \geqslant j}^{N} u_{i j k}^{p} R_{i} S_{j} S_{k}, \\
Y_{p}= & S_{p}+\sum_{i=1}^{N} \sum_{j=1}^{N} \gamma_{i j}^{p} R_{i} S_{j}+\sum_{i=1}^{N} \sum_{j \geqslant i}^{N} \sum_{k \geqslant j}^{N} \mu_{i j k}^{p} S_{i} S_{j} S_{k} \\
& +\sum_{i=1}^{N} \sum_{j=1}^{N} \sum_{k \geqslant j}^{N} v_{i j k}^{p} S_{i} R_{j} R_{k} .
\end{aligned}
$$

The new-defined variables, $R_{p}$ and $S_{p}$, are respectively homogeneous to a displacement and a velocity. They are called the normal co-ordinates. The coefficients of this non-linear change of variables $\left(a_{i j}^{p}, b_{i j}^{p}, r_{i j k}^{p}, u_{i j k}^{p}, \gamma_{i j}^{p}, \mu_{i j k}^{p}, v_{i j k}^{p}\right)$ are given in Appendix A, the complete proof leading to Eqs. (4) is given in Ref. [32].

Substituting for Eqs. (4) in Eqs. (2) gives the complete dynamics for the $N$ oscillators, where all invariant-breaking terms have been cancelled:

$$
\begin{gathered}
\forall p=1, \ldots, N: \quad \dot{R}_{p}=S_{p}, \\
\dot{S}_{p}=-\omega_{p}^{2} R_{p}-\left(A_{p p p}^{p}+h_{p p p}^{p}\right) R_{p}^{3}-B_{p p p}^{p} R_{p} S_{p}^{2}-R_{p}\left[\sum_{j>p}^{N}\left[\left(A_{j p j}^{p}+A_{p j j}^{p}+h_{p j j}^{p}\right) R_{j}^{2}+B_{p j j}^{p} S_{j}^{2}\right]\right. \\
\left.+\sum_{i<p}\left[\left(A_{i i p}^{p}+A_{p i i}^{p}+h_{i i p}^{p}\right) R_{i}^{2}+B_{p i i}^{p} S_{i}^{2}\right]\right]-S_{p}\left[\sum_{j>p}^{N} B_{j p j}^{p} R_{j} S_{j}+\sum_{i<p} B_{i i p}^{p} R_{i} S_{i}\right]
\end{gathered}
$$

The coefficients $\left(A_{i j k}^{p}, B_{i j k}^{p}\right)$ arise from the cancellation of the quadratic terms. Their expressions are

$$
\begin{gathered}
A_{i j k}^{p}=\sum_{l \geqslant i}^{N} g_{i l}^{p} a_{j k}^{l}+\sum_{l \leqslant i} g_{l i}^{p} a_{j k}^{l}, \\
B_{i j k}^{p}=\sum_{l \geqslant i}^{N} g_{i l}^{p} b_{j k}^{l}+\sum_{l \leqslant i} g_{l i}^{p} b_{j k}^{l} .
\end{gathered}
$$

The following remarks are important here:

- In Eqs. (5), all invariant-breaking terms have been cancelled. Hence the invariance property is recovered.

- Eq. (4) is identity-tangent: the linear results are thus recovered for small amplitudes.

- Velocity-dependent terms arise in Eqs. (5) only if quadratic non-linearity is present in the initial problem given by (2). For example, for a non-linear beam problem, for which $g_{i j}^{p} \equiv 0$, no 
velocity-dependent terms appear when considering the third order approximation of the dynamics onto invariant manifolds.

- Normal form (5) can be written with the knowledge of the linear eigenspectrum only [20,21]. Hence, ex-nihilo models can be written to study the dynamics onto the manifolds (see e.g. Ref. [35]). Then, one is able to recover the modal co-ordinates with Eqs. (4). The operation stated here bears a resemblance with modal decomposition usually performed at the linear stage, except that the space is now curved; and thus superposition is not straightforward.

\subsection{Internal resonance}

Internal resonances have been neglected in the previous section. However, considering an internal resonance in the system does not lead to tedious calculations for computing the nonlinear change of co-ordinates. This is an important feature since usual methods proposed in the literature to compute the NNMs, which rely upon the centre manifold reduction theorem $[10,11,36]$, or upon asymptotic development in the PDE $[8,16,27]$, become very complicated in case of internal resonance.

An internal resonance appears with the failure of the calculation of one coefficient in Eq. (4). As is usual in this case, the affected coefficient has just to be set to zero, and the associated monomial stays in the normal form [20,32], as has already been done with the trivially resonant cubic terms.

\subsection{Single-mode motion}

In this section, the dynamical equations, that are related to the motion along a single non-linear mode, are derived. The results presented here are important because a physically observed singlemode motion occurs on an invariant manifold and, at the non-linear stage, has to be related to a non-linear normal mode motion. It is the aim of the NNMs computations to offer reduced order models, described by a limited number of variables, which hold the important properties exhibited by the non-linearity. This is achieved through invariant manifolds and will be illustrated with the hardening (or softening) behaviour of the oscillations.

To investigate the $p$ th NNM, one has just to cancel all other variables since the bending of the phase space is contained within the non-linear change of co-ordinates:

$$
\forall k \neq p: R_{k}=S_{k}=0 .
$$

Substituting for Eq. (7) in Eq. (4) gives the geometry of the manifold in phase space:

$$
\begin{gathered}
\forall k \neq p: X_{k}=a_{p p}^{k} R_{p}^{2}+b_{p p}^{k} S_{p}^{2}+r_{p p p}^{k} R_{p}^{3}+u_{p p p}^{k} R_{p} S_{p}^{2}, \\
Y_{k}=\gamma_{p p}^{k} R_{p} S_{p}+\mu_{p p p}^{k} S_{p}^{3}+v_{p p p}^{k} S_{p} R_{p}^{2}
\end{gathered}
$$

This third order approximation of the invariant manifold has been compared with precedent calculations led by other authors $[28,16]$, and all coefficients match.

The dynamics onto the $p$ th manifold is found by substituting for Eq. (7) in Eq. (5):

$$
\ddot{R}_{p}+\omega_{p}^{2} R_{p}+\left(A_{p p p}^{p}+h_{p p p}^{p}\right) R_{p}^{3}+B_{p p p}^{p} R_{p} \dot{R}_{p}^{2}=0 .
$$

This equation has to be compared to the usual single linear mode Galerkin approximation which is of current use for the analysis of non-linear oscillations of continuous systems. Performing a 
single linear mode approximation means projecting the PDE onto the $p$ th linear eigenspace. It is found by setting: $\forall k \neq p: X_{k}=0$ in Eq. (2). Then the dynamics is governed by

$$
\ddot{X}_{p}+\omega_{p}^{2} X_{p}+g_{p p}^{p} X_{p}^{2}+h_{p p p}^{p} X_{p}^{3}=0 .
$$

This procedure can lead to erroneous results because the linear eigenspace is not invariant. Taking the bending of the phase space into account can drastically change the behaviour. This will be underlined by deriving the amplitude-frequency relation, usually known as the backbone curve. Using any of the perturbation methods available, one can find, at first order and for Eq. (9):

$$
\left.\omega_{\mathrm{NL}}=\omega_{p} \quad 1+\frac{3\left(A_{p p p}^{p}+h_{p p p}^{p}\right)+\omega_{p}^{2} B_{p p p}^{p}}{8 \omega_{p}^{2}} a^{2}\right)
$$

where $\omega_{\mathrm{NL}}$ is the non-linear angular frequency, which depends on the amplitude $a$ considered.

The hardening or softening behaviour for a single non-linear mode motion is governed by the sign of the term

$$
\Gamma_{p}=\frac{3\left(A_{p p p}^{p}+h_{p p p}^{p}\right)+\omega_{p}^{2} B_{p p p}^{p}}{8 \omega_{p}^{2}} .
$$

One can see that the influences of all the linear modes are taken into account through the terms $A_{p p p}^{p}$ and $B_{p p p}^{p}$. This will give a greater accuracy than considering only a single linear mode. It has to be compared to the coefficient $\tilde{\Gamma}_{p}$ which dictates the behaviour of the non-linear oscillations for Eq. (10), which reads

$$
\left.\tilde{\Gamma}_{p}=\frac{1}{8 \omega_{p}^{2}} 3 h_{p p p}^{p}-\frac{10 g_{p p}^{p 2}}{3 \omega_{p}^{2}}\right) .
$$

The remainder of this article is devoted to the processing of two examples, where the precedent theoretical results will be illustrated. A particular emphasis will be put on the erroneous results one can find when applying a single-mode Galerkin reduction.

\section{Example 1: a discrete system}

\subsection{Equations of motion}

The first example is devoted to a discrete two d.o.f. system, composed of a mass $m$ connected to a frame by two geometrically non-linear springs (Fig. 2). The potential energy of spring $i(i \in\{1,2\})$ is assumed to be

$$
\mathscr{E}_{p i}=\frac{1}{2} k_{i} l_{0}^{2} e_{i}^{2}, \quad \text { with } \quad e_{i}=\frac{1}{2} \frac{l_{i}^{2}-l_{0}^{2}}{l_{0}^{2}} .
$$

$k_{i}$ is the linear stiffness of spring $i$, and $l_{i}$ is its deformed length. The tension in the springs is then

$$
N_{i}=-\frac{\partial \mathscr{E}_{p i}}{\partial l_{i}}=-\frac{1}{2} k_{i} l_{0}\left[\left(\frac{l_{i}}{l_{0}}\right)^{3}-\frac{l_{i}}{l_{0}}\right]=-k_{i}\left[\Delta l_{i}+\frac{3}{2} \frac{\Delta l_{i}^{2}}{l_{0}}+\frac{1}{2} \frac{\Delta l_{i}^{3}}{l_{0}^{2}}\right],
$$




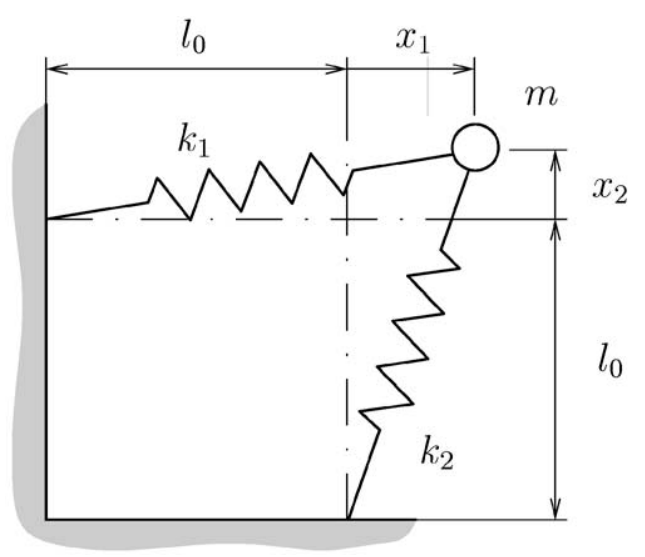

Fig. 2. The physical two d.o.f. system for example 1 .

with $\Delta l_{i}=l_{i}-l_{0}$. One can notice that the springs have quadratic and cubic non-linearities. Applying Lagrange's equations to the mass gives the non-linear ODEs which govern the motion:

$$
\mathscr{L}_{i}:\left(\frac{\mathrm{d}}{\mathrm{d} t} \frac{\partial}{\partial \dot{x}_{i}}-\frac{\partial}{\partial x_{i}}\right)\left(\mathscr{E}_{k}+\mathscr{E}_{p}\right)=0
$$

with $\mathscr{E}_{k}$ denoting the kinetic energy of mass $m$, and $\mathscr{E}_{p}=\mathscr{E}_{p 1}+\mathscr{E}_{p 2}$ the total potential energy. It results in

$$
\begin{aligned}
& \ddot{X}_{1}+\omega_{1}^{2} X_{1}+\frac{\omega_{1}^{2}}{2}\left(3 X_{1}^{2}+X_{2}^{2}\right)+\omega_{2}^{2} X_{1} X_{2}+\frac{\omega_{1}^{2}+\omega_{2}^{2}}{2} X_{1}\left(X_{1}^{2}+X_{2}^{2}\right)=0, \\
& \ddot{X}_{2}+\omega_{2}^{2} X_{2}+\frac{\omega_{2}^{2}}{2}\left(3 X_{2}^{2}+X_{1}^{2}\right)+\omega_{1}^{2} X_{1} X_{2}+\frac{\omega_{1}^{2}+\omega_{2}^{2}}{2} X_{2}\left(X_{1}^{2}+X_{2}^{2}\right)=0,
\end{aligned}
$$

$X_{1}=x_{1} / l_{0}$ and $X_{2}=x_{2} / l_{0}$ are dimensionless displacements defining the position of the mass, $\omega_{1}^{2}=k_{1} / m$ and $\omega_{2}^{2}=k_{2} / m$ are the natural frequencies of the system. The system is naturally linearly uncoupled, as the two linear modes of motion are $X_{1}$ and $X_{2}$. It is a consequence of the orthogonal configuration of the two springs at rest.

\subsection{Regions of hardening and softening behaviour}

In this subsection, a detailed comparison between the motion along the first linear mode and along the first non-linear mode is provided. It is assumed that no internal resonance exists between the two modes.

Considering the first linear mode leads to a dynamics governed by

$$
\ddot{X}_{1}+\omega_{1}^{2} X_{1}+\frac{3 \omega_{1}^{2}}{2} X_{1}^{2}+\frac{\omega_{1}^{2}+\omega_{2}^{2}}{2} X_{1}^{3}=0 .
$$

The backbone curve in this case is given by

$$
\tilde{\omega}_{\mathrm{NL}}=\omega_{1}\left(1+\left(-\frac{3}{4}+\frac{3 \omega_{2}^{2}}{16 \omega_{1}^{2}}\right) a^{2}\right),
$$


where $\tilde{\omega}_{\mathrm{NL}}$ stands for the non-linear angular frequency found with a linear mode approximation, and $a$ is the amplitude of the motion considered: $X_{1}=a \cos \left(\tilde{\omega}_{\mathrm{NL}} t+\beta_{0}\right)+\cdots$.

In the parameter plane $\left(\omega_{1}, \omega_{2}\right)$, regions of hardening or softening behaviour are governed by the sign of

$$
\tilde{\Gamma}_{1}=-\frac{3}{4}+\frac{3 \omega_{2}^{2}}{16 \omega_{1}^{2}}
$$

Considering now the first non-linear mode, which is the right approximation if one is interested in a motion non-linearly vibrating along the first physical mode, indicates that the oscillations are governed by Eq. (9), with $p=1$. Computing the coefficients and replacing in Eq. (12) shows that the hardening or softening behaviour onto the first invariant manifold is determined by the sign of

$$
\Gamma_{1}=-\frac{3}{4}+\frac{3 \omega_{2}^{2}}{16 \omega_{1}^{2}}+\frac{\omega_{2}^{2}\left(8 \omega_{1}^{2}-3 \omega_{2}^{2}\right)}{16 \omega_{1}^{2}\left(\omega_{2}^{2}-4 \omega_{1}^{2}\right)} .
$$

It appears clearly that considering the bending of the phase space caused by the presence of the second oscillator gives rise to a correction on the backbone curve. More specifically, this effect can be drastic and can change the effective behaviour of the non-linear oscillations. Fig. 3 shows the hardening and softening region in the two cases (simply given by the signs of $\tilde{\Gamma}_{1}$ in the linear case, and $\Gamma_{1}$ in the non-linear case). One can notice for example the upper-left region, which is predicted to exhibit a hardening behaviour by the linear approximation, whereas the real behaviour is soft. Hence single linear mode approximation can give results that are substantially wrong. In the next subsection, numerical simulations validate these results.

\subsection{Numerical simulations}

Numerical simulations have been performed on system (17) in order to verify the precedent theoretical results. First, a graphical representation of the first invariant manifold is shown in Fig. 4. The third order approximation of the invariant manifold has been calculated with Eqs. (8).
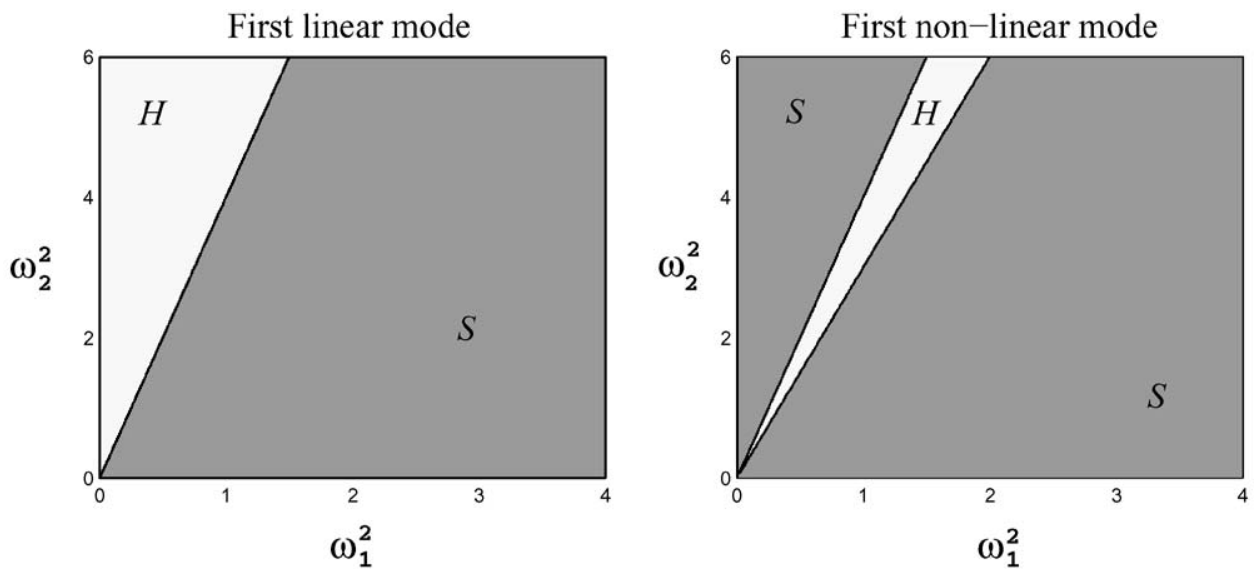

Fig. 3. Regions of hardening $(H)$ and softening $(S)$ behaviour for Example 1. Left: first linear mode approximation. Right: first non-linear mode. 

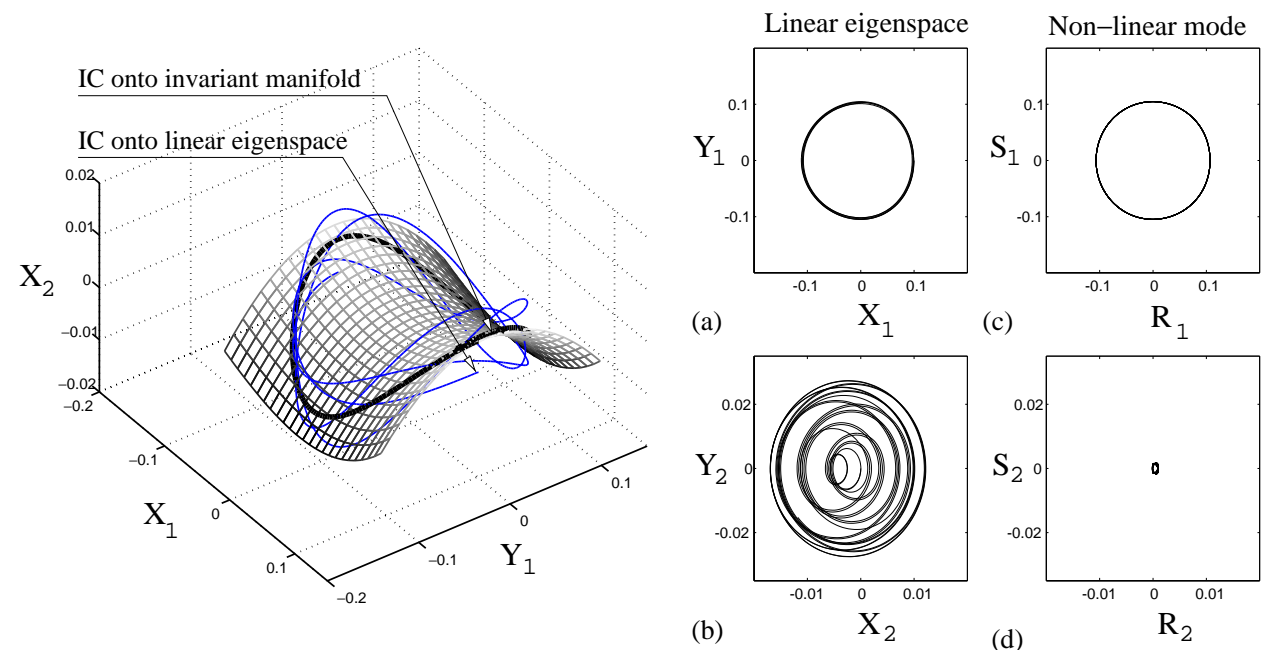

Fig. 4. Representation of the first invariant manifold with $\omega_{1}=1$ and $\omega_{2}=\sqrt{3}$. Left: the surface represents the first NNM. Two trajectories have been represented with two different initial conditions (IC). Solid line: IC onto linear eigenspace. Heavy line: IC onto the first NNM. Right: projections of the two trajectories onto their respective subspaces. (a)-(b): Orbit initiated along the linear mode, projected onto the linear eigenspaces. (c)-(d): Orbit initiated along the first NNM, projected onto the two invariant manifolds.

Two orbits have been represented. The first one has for the initial condition a point which relies on the linear eigenspace. One can see that it gives rise to a whirling trajectory. The vertical displacement corresponds to the quantity that is neglected when one performs a single linear mode approximation. This is highlighted on the projections: Fig. 4(b) shows exactly the neglected quantity. For the second trajectory, the initial condition has been taken onto the invariant manifold. The real position has been found numerically by a shooting technique, which has also been utilized in Fig. 5 (second row). The neglected quantity is divided by 15. The drawback of considering only a third order approximation for the invariant manifolds can be numerically estimated on this example. Taking the initial condition onto the third order approximation of the first NNM leads to a residual contribution which is divided by seven compared to the linear case.

A second set of simulations has been conducted in order to compute numerical backbone curves, and to compare them with the analytical first order development. Fig. 5 shows the results obtained for three different cases, corresponding to the three regions highlighted in Fig. 3. For the linear-mode approximation, Eq. (18) has been used. For computing the backbone curve onto the invariant manifold, the whole system (Eqs. (17)) has been used, and the initial condition for the computed trajectory has been taken onto the first NNM, for each amplitude. The second row of Fig. 5 shows the positions of the two invariant manifolds in space $\left(X_{1}, X_{2}\right)$. One can see that for too large amplitudes, the divergence between the third order approximation of the invariant manifolds and the real position becomes noticeable.

Through this example, it has been shown that a linear single-mode approximation can lead to wrong results when predicting hardening or softening behaviour. These errors are corrected when one considers the NNMs, which are the right approximation, at the non-linear stage, for a singlemode motion. The next section examines the same features on a continuous system. 

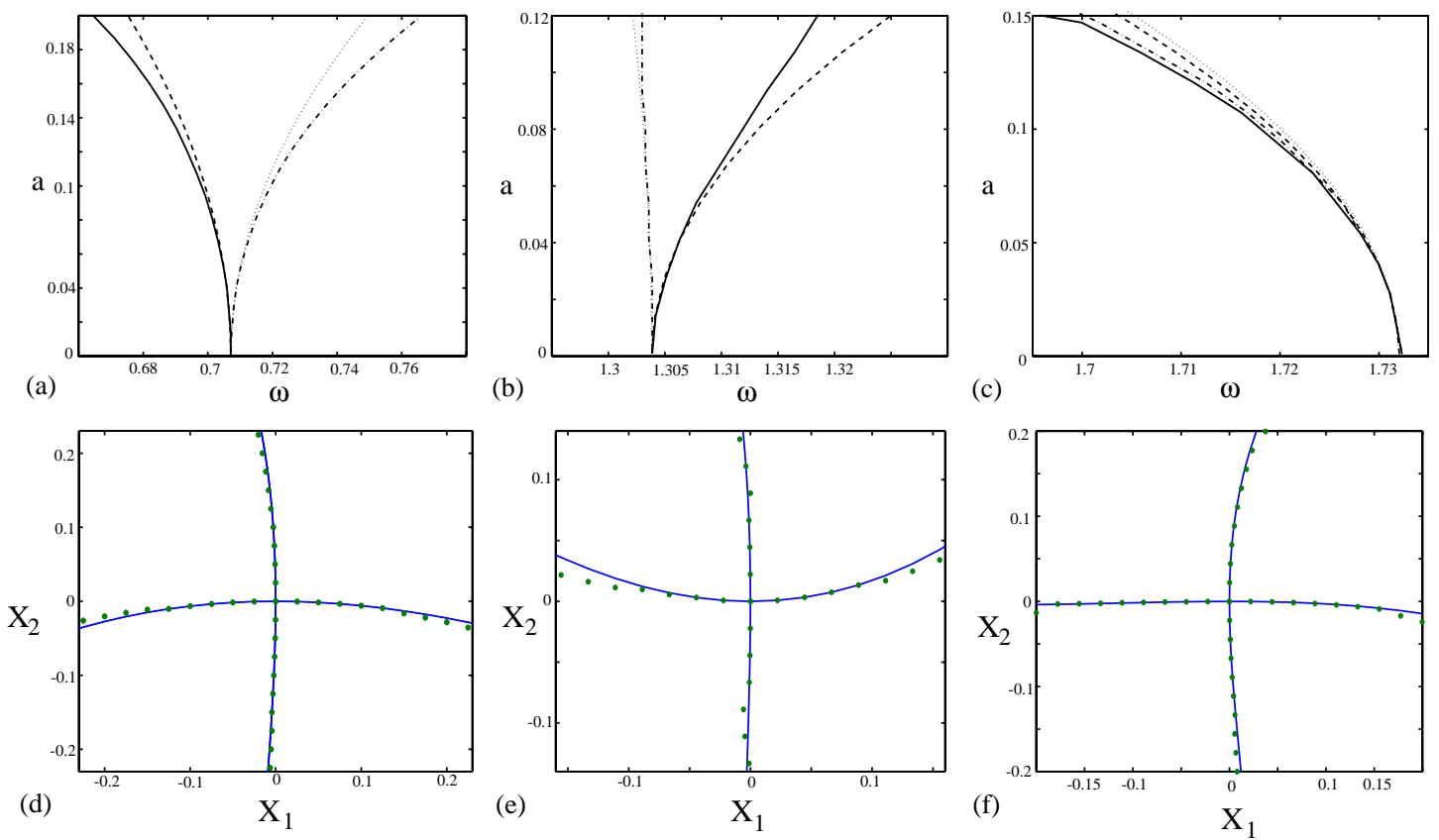

Fig. 5. First row: Comparison of analytical and backbone curves for example 1, first mode. The reference solution is given by numerically computing the amplitude/frequency relation for an initial condition onto the first invariant manifold (solid heavy line). Dashed line (- - -): first order analytical backbone curve onto the first NNM (governed by $\Gamma_{1}$, cf. Eq. (11)). Dash-dotted line (- - - - ): numerical backbone curve onto the first linear eigenspace. Dotted line (......): first order analytical backbone curve onto the first linear mode. Second row: positions of the two manifolds in space $\left(X_{1}, X_{2}\right)$. Solid line: third order approximation; $(*)$ : real position. (a)-(d) $\omega_{1}=\sqrt{0.5}, \omega_{2}=\sqrt{6}$; (b)-(e): $\omega_{1}=$ $\sqrt{1.7}, \omega_{2}=\sqrt{6} ;$ (c)-(f): $\omega_{1}=\sqrt{3}, \omega_{2}=1$.

\section{Example 2: a continuous system}

\subsection{Equations of motion}

A linear hinged-hinged beam resting on a non-linear elastic foundation with distributed quadratic and cubic non-linearities is considered. This example has already been studied in Refs. $[5,8]$, and it is the aim of this section to compare their results with the methodology proposed herein, based on invariant manifolds and normal form theory.

In non-dimensional form, the undamped transverse vibrations are governed by $[5,8]$

$$
\frac{\partial^{2} w}{\partial t^{2}}+\frac{\partial^{4} w}{\partial x^{4}}+\alpha_{2} w^{2}+\alpha_{3} w^{3}=0
$$

with boundary conditions

$$
w(x, t)=0, \quad \frac{\partial^{2} w(x, t)}{\partial x^{2}}=0 \quad \text { for } x=0,1 .
$$


$w(x, t)$ is the transverse displacement, $\alpha_{2}$ and $\alpha_{3}$ are constants. The linear analysis provides the eigenmodes as well as the eigenfrequencies:

$$
\begin{gathered}
\Phi_{n}(x)=\sqrt{2} \sin (n \pi x), \\
\omega_{n}=n^{2} \pi^{2} .
\end{gathered}
$$

Projection onto the linear mode basis is performed via the development:

$$
w(x, t)=\sum_{p=1}^{N} X_{p}(t) \Phi_{p}(x)
$$

which is inserted into Eq. (22). This leads to the following temporal problem: $\forall p=1, \ldots, N$ :

$$
\ddot{X}_{p}+\omega_{p}^{2} X_{p}+\sum_{i, j=1}^{N} g_{i j}^{p} X_{i} X_{j}+\sum_{i, j, k=1}^{N} h_{i j k}^{p} X_{i} X_{j} X_{k}=0
$$

where

$$
g_{i j}^{p}=\alpha_{2} \int_{0}^{1} \Phi_{i}(x) \Phi_{j}(x) \Phi_{p}(x) \mathrm{d} x, \quad h_{i j k}^{p}=\alpha_{3} \int_{0}^{1} \Phi_{i}(x) \Phi_{j}(x) \Phi_{k}(x) \Phi_{p}(x) \mathrm{d} x .
$$

\subsection{Internal resonances and invariance}

Normal form for Eq. (27) is computed with the results presented in Section 1. As a consequence of the values of the eigenfrequencies, Eq. (25), the system exhibits many internal resonances. They are listed in Table 1, for the 10 first eigenfrequencies. Higher-dimensional invariant manifolds should be considered to study the dynamics, as strong coupling (resonant) terms arise from the internal resonance relations. But, as it is explained next, single-mode motion on two-dimensional invariant manifolds exists for the linear beam resting on a non-linear elastic foundation, because in this problem, all resonant coupling terms are not invariant-breaking.

Table 1

Internal resonance relations between the ten first eigenfrequencies

Order-two internal resonances

$\omega_{5}=\omega_{3}+\omega_{4}$

$\omega_{10}=\omega_{6}+\omega_{8}$

Order-three internal resonances

$\omega_{1}=\omega_{4}+\omega_{7}-\omega_{8}$

$\omega_{1}=2 \omega_{5}-\omega_{7}$

$\omega_{2}=\omega_{6}+\omega_{7}-\omega_{9}$

$\omega_{3}=\omega_{1}+2 \omega_{2}$

$\omega_{6}=\omega_{2}+2 \omega_{4}$

$\omega_{7}=\omega_{2}+\omega_{3}+\omega_{6}$

$\omega_{9}=\omega_{1}+\omega_{8}+\omega_{4}$

$\omega_{9}=\omega_{3}+2 \omega_{6}$

$\omega_{9}=2 \omega_{4}+\omega_{7}$ 
An internal resonance of the form: $\omega_{p}=2 \omega_{k}$ (second order), or $\omega_{p}=3 \omega_{k}$ (third order) creates a monom which breaks the invariance of two-dimensional manifolds. For example, the relationship $\omega_{p}=2 \omega_{k}$ implies the presence of an unremovable monom $R_{k}^{2}$ in the evolution equation for $R_{p}$. Hence letting $R_{p}=0$ to study the $k$ th NNM alone is not possible because of this "source" term in the $p$ th equation. In this particular case, a four-dimensional manifold with the NNMs $p$ and $k$ has to be considered.

On the other hand, internal resonance relations which strictly imply more than two eigenfrequencies do not give rise to invariant-breaking terms, since it is always possible to cancel the co-ordinates to study the dynamics onto a single two-dimensional manifold. This remark should be compared with the absence of a stable single-mode solution in a perturbative study of the dynamics of coupled oscillators with invariant-breaking terms (see e.g. Refs. [37,1]); or its presence when only resonant terms that do not break the invariance are involved in the equations (see e.g. Refs. $[38,39,1])$.

\subsection{Hardening/softening behaviour}

The dynamics onto a single NNM is now studied. Attention will be focused on the first three eigenmodes. For the $p$ th NNM, the oscillations are governed by Eq. (9), and the hardening or softening behaviour by the sign of $\Gamma_{p}$ (Eq. (12)). Table 2 gives the values of $\Gamma_{p}$ for $p=1,2,3$, and for an increasing value $N$ of linear modes retained in the analysis. The coefficients $\alpha_{2}$ and $\alpha_{3}$ have been set, respectively, to 12 and 1 for this computation.

One can notice that taking the NNMs into account leads to a change of sign for $\Gamma_{p}$, for modes 2 and 3. Hence a linear-mode Galerkin approximation fails to predict the correct behaviour of the oscillations. The convergence of $\Gamma_{p}$ for the first three modes is obtained here, with 5 significant digits of accuracy, for $N=8$. In the following, the number of retained linear modes will thus be chosen equal to 10 to get confidence in the accuracy of the results.

Fig. 6 shows (first row) the hardening and softening regions in the parameter plane $\left(\alpha_{2}, \alpha_{3}\right)$. In Fig. 6(a), for the first mode, the two regions (given, respectively, by the linear approximation and the NNM) are identical. Considering the NNM does not change the global behaviour substantially. This is not the case for modes 2 and 3. For mode 2, Fig. 6(b), the linear approximation predicts a hardening behaviour in all the plane, since the quadratic coefficient is equal to zero: $g_{22}^{2}=0$. Considering the second NNM shows that the correct result is different and that a softening region is present. The second and third rows show the backbone curves

\section{Table 2}

Convergence of the value of $\Gamma_{p}$, which determines the hardening or softening behaviour of the mode considered, with increasing $N$. The first line gives the value of $\tilde{\Gamma}_{p}$ (single linear mode approximation). NB.: the value of $\Gamma_{3}$ with two modes have been computed with modes 1 and $3 . \alpha_{2}=12$ and $\alpha_{3}=1$

\begin{tabular}{lllr}
\hline$N$ & $\Gamma_{1}$ & $\Gamma_{2}$ & \multicolumn{1}{c}{$\Gamma_{3}$} \\
\hline 1 & $-3.338 \mathrm{e}-3$ & $36.091 \mathrm{e}-5$ & $7.113 \mathrm{e}-5$ \\
2 & $-3.338 \mathrm{e}-3$ & $-7.299 \mathrm{e}-5$ & $-0.907 \mathrm{e}-5$ \\
3 & $-3.346 \mathrm{e}-3$ & $-8.231 \mathrm{e}-5$ & $-0.907 \mathrm{e}-5$ \\
5 & $-3.346 \mathrm{e}-3$ & $-8.243 \mathrm{e}-5$ & $-0.918 \mathrm{e}-5$ \\
8 & $-3.346 \mathrm{e}-3$ & $-8.243 \mathrm{e}-5$ & $-0.918 \mathrm{e}-5$ \\
\hline
\end{tabular}





Fig. 6. First row: hardening and softening regions using the linear mode approximation (dashed lines, $h / s$ ) and the nonlinear mode approximation (solid lines, $H / S$ ) for the first three modes. (d)-(i) Backbone curves in different cases, solid line:NNM, dash-dot: linear mode. (d) $\alpha_{2}=12, \alpha_{3}=1$. (g) $\alpha_{2}=5, \alpha_{3}=2$. (e) $\alpha_{2}=12, \alpha_{3}=0.5$. (h) $\alpha_{2}=5, \alpha_{3}=2$. (f) $\alpha_{2}=12, \alpha_{3}=0.5$. (i) $\alpha_{2}=1, \alpha_{3}=0.01$.

analytically obtained, respectively, onto the linear eigenspace and onto the invariant manifold, and for different values of $\alpha_{2}$ and $\alpha_{3}$.

These results have been compared with those derived in Refs. [5,8], where a perturbation technique (the method of multiple scales) is used directly into the PDE (22). The hardening and softening regions are exactly the same, showing that the methods are equivalent for determining the behaviour of the non-linear oscillations.

\subsection{Drift and mode shapes}

Using a first order perturbation technique, one can find that the solution of Eq. (9), governing the oscillations onto the manifold is given by

$$
R_{p}=a_{0} \cos \left(\omega_{\mathrm{NL}} t+\beta_{0}\right)+\cdots
$$

with $\omega_{\mathrm{NL}}=\omega_{p}\left(1+\Gamma_{p} a_{0}^{2}\right)$; for an amplitude $a_{0}$.

Substituting for Eq. (29) into the non-linear change of co-ordinates gives the solution with the modal co-ordinates. Investigating the NNM labelled $p$, one has to replace with

$$
\forall k \neq p: X_{k}=a_{p p}^{k} R_{p}^{2}+b_{p p}^{k} S_{p}^{2}+r_{p p p}^{k} R_{p}^{3}+u_{p p p}^{k} R_{p} S_{p}^{2},
$$




$$
k=p: X_{p}=R_{p}+a_{p p}^{p} R_{p}^{2}+b_{p p}^{p} S_{p}^{2}
$$

into

$$
w_{p}(x, t)=X_{p}(t) \Phi_{p}(x)+\sum_{k \neq p} X_{k}(t) \Phi_{k}(x)
$$

To obtain the complete solution for the displacement. $w_{p}(x, t)$ is written for a displacement along the $p$ th NNM. Omitting the cubic terms in the precedent equations to get a second order solution, gives

$$
\begin{aligned}
w_{p}(x, t)= & a_{0} \cos \left(\omega_{\mathrm{NL}} t+\beta_{0}\right) \Phi_{p}(x) \\
& +\sum_{k=1}^{N}\left[a_{p p}^{k} \frac{a_{0}^{2}}{2}+b_{p p}^{k} \frac{a_{0}^{2} \omega_{\mathrm{NL}}^{2}}{2}+\left(a_{p p}^{k} \frac{a_{0}^{2}}{2}-b_{p p}^{k} \frac{a_{0}^{2} \omega_{\mathrm{NL}}^{2}}{2}\right) \cos \left(2 \omega_{\mathrm{NL}} t+2 \beta_{0}\right)\right] \Phi_{k}(x) .
\end{aligned}
$$

Hence the quadratic non-linearity gives rise to a drift in the oscillations, which is written here as

$$
d_{p}(x, t)=\sum_{k=1}^{N}\left[a_{p p}^{k} \frac{a_{0}^{2}}{2}+b_{p p}^{k} \frac{a_{0}^{2} \omega_{\mathrm{NL}}^{2}}{2}\right] \Phi_{k}(x) .
$$

It has to be compared with the same drift predicted with a linear-mode Galerkin approximation, which reads $[5,8]$

$$
\tilde{d}_{p}(x, t)=-\frac{\delta_{p} \alpha_{2}}{2 \omega_{p}^{2}} a_{0}^{2} \Phi_{p}(x)
$$

where $\delta_{p}=(4 \sqrt{2} /(3 p \pi))(1-\cos p \pi)$.

Mode shapes as well as drifts have been compared for the three methods: linear (single-mode) approximation, Non-linear single-mode motion and perturbation method directly in the PDE [5,8]. The normalized results for modes 2 and 3 are shown in Fig. 7. For the mode shapes, the

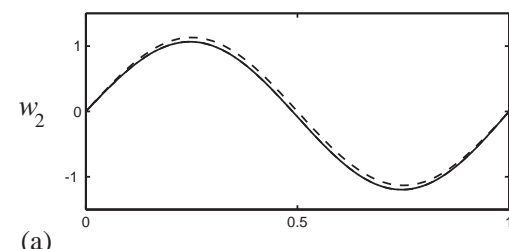

(a)

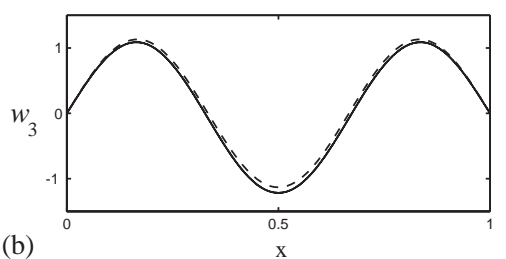

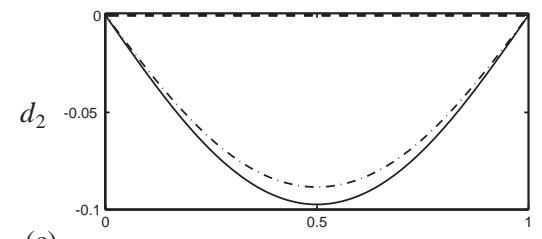

(c)

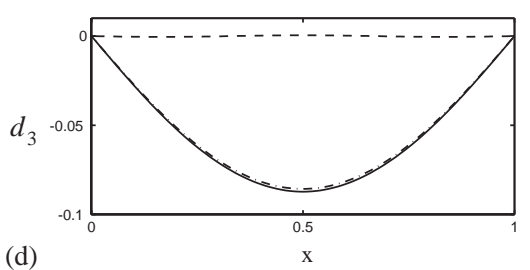

Fig. 7. Mode shapes (left column) and drifts (right) for mode 2 (first row) and 3 (second row). Solid line: NNM solution. Dash-dotted line: solution of Nayfeh and Lacarbonara, using a perturbation method directly into the PDE. Dashed line: linear solution. 
method used by Nayfeh and Lacarbonara gives the same results as in this study. For the drifts, a little difference is observed for mode 2 .

\section{Conclusion}

In this paper, normal form theory has been used in order to define non-linear normal modes. Keeping an oscillator form at linear stage (which is realized with two-dimensional real nondiagonal blocks in the linear part of the dynamics) allows one to have real equations throughout the calculations. This is contrary to the usual manner in which normal form has always been presented in vibration theory, and allows precise comparison of this method with others, already developed to define NNMs. Moreover, a clean mathematical framework to define non-linear modal analysis/synthesis is given. Indeed, the non-linear change of co-ordinates can be viewed as an extension of the linear modal method.

Analysis should be performed with the help of normal form, since it allows derivation of reduced order models that capture the essential of the dynamics, and express amplitudedependent mode shapes. Moreover, the question of a clever truncature should be asked after the non-linear change of co-ordinates. Synthesis is simplified since the dynamics onto the invariant manifolds is simply given by the normal form of the vibratory problem, and normal form could be written with the knowledge of the linear eigenspectrum only.

Throughout the calculations, third order asymptotic developments have been used. This constitutes the main limitation of the method. However, it enables derivation of important results, which have been underlined on simple examples. Single NNM motions have been studied, showing that it allows prediction of the effective trend of the non-linearity for structural systems (hardening/softening behaviour). It has been contrasted with the single linear mode Galerkin approximation which is of current use, although it can lead to erroneous results. The role of the quadratic non-linearity has been underlined. The second example allowed comparison of the NNM results with a method proposed in Refs. [5,8] in order to overcome the problems caused by the linear truncature. It has been shown that the two methods yield the same results.

\section{Acknowledgements}

The authors would like to thank Bruno Cochelin from LMA-Marseille for suggesting the first example. Paul Manneville is also thanked for his lessons on dynamical systems theory.

\section{Appendix A. Expressions of the coefficients for Eqs. (4)}

Expressions of the coefficients $\left(a_{i j}^{p}, b_{i j}^{p}, r_{i j k}^{p}, u_{i j k}^{p}, \gamma_{i j}^{p}, \mu_{i j k}^{p}, v_{i j k}^{p}\right)$ used in Eqs. (4), which allows one to go from the modal variables (associated with the orthogonal linear grid) to the normal co-ordinates (associated to the invariant manifold and the curved grid) are given here. 


\section{A.1. Quadratic coefficients}

The following expressions are obtained, $\forall p=1, \ldots, N$ :

$$
\begin{gathered}
\forall i=1, \ldots, N, \forall j \geqslant i, \ldots, N: \\
a_{i j}^{p}=\frac{\omega_{i}^{2}+\omega_{j}^{2}-\omega_{p}^{2}}{D_{i j p}} g_{i j}^{p}, \\
b_{i j}^{p}=\frac{2}{D_{i j p}} g_{i j}^{p}, \\
c_{i j}^{p}=0, \\
\alpha_{i j}^{p}=0, \\
\beta_{i j}^{p}=0, \\
\gamma_{i i}^{p}=\frac{2}{4 \omega_{i}^{2}-\omega_{p}^{2}} g_{i i}^{p}, \\
\forall i=1, \ldots, N, \quad \forall j>i, \ldots, N: \\
\gamma_{i j}^{p}=\frac{\omega_{j}^{2}-\omega_{i}^{2}-\omega_{p}^{2}}{D_{i j p}} g_{i j}^{p}, \\
\gamma_{j i}^{p}=\frac{\omega_{i}^{2}-\omega_{j}^{2}-\omega_{p}^{2}}{D_{i j p}} g_{i j}^{p},
\end{gathered}
$$

where $D_{i j p}=\left(\omega_{i}+\omega_{j}-\omega_{p}\right)\left(\omega_{i}+\omega_{j}+\omega_{p}\right)\left(-\omega_{j}+\omega_{i}+\omega_{p}\right)\left(\omega_{i}-\omega_{j}-\omega_{p}\right)$.

\section{A.2. Cubic coefficients}

The following coefficients, which correspond to the trivially resonant terms, are equal to zero:

$$
\begin{aligned}
& \forall p=1, \ldots, N: \quad u_{p p p}^{p}=r_{p p p}^{p}=\mu_{p p p}^{p}=v_{p p p}^{p}=0, \\
& \forall j>p, \ldots, N: \quad r_{p j j}^{p}=u_{p j j}^{p}=u_{j p j}^{p}=0, \\
& \mu_{p j j}^{p}=v_{p j j}^{p}=v_{j p j}^{p}=0, \\
& \forall i<p: \quad r_{i i p}^{p}=u_{i i p}^{p}=u_{p i i}^{p}=0, \\
& \mu_{i i p}^{p}=v_{i i p}^{p}=v_{p i i}^{p}=0 .
\end{aligned}
$$

The non-zero coefficients are now given, $\forall p=1, \ldots, N$ :

$$
\begin{gathered}
\forall i=1, \ldots, N, i \neq p: \\
r_{i i i}^{p}=\frac{1}{D_{i p}^{(1)}}\left[\left(7 \omega_{i}^{2}-\omega_{p}^{2}\right)\left(h_{i i i}^{p}+A_{i i i}^{p}\right)+2 \omega_{i}^{4} B_{i i i}^{p}\right],
\end{gathered}
$$




$$
\begin{gathered}
u_{i i i}^{p}=\frac{1}{D_{i p}^{(1)}}\left[6 h_{i i i}^{p}+6 A_{i i i}^{p}+\left(3 \omega_{i}^{2}-\omega_{p}^{2}\right) B_{i i i}^{p}\right], \\
\mu_{i i i}^{p}=u_{i i i}^{p}, \\
v_{i i i}^{p}=\frac{1}{D_{i p}^{(1)}}\left[\left(9 \omega_{i}^{2}-3 \omega_{p}^{2}\right)\left(h_{i i i}^{p}+A_{i i i}^{p}\right)+2 \omega_{p}^{2} \omega_{i}^{2} B_{i i i}^{p}\right],
\end{gathered}
$$

where $D_{i p}^{(1)}=\left(\omega_{p}^{2}-\omega_{i}^{2}\right)\left(\omega_{p}^{2}-9 \omega_{i}^{2}\right)$.

$$
\begin{aligned}
& \forall i=1, \ldots, N-1, i \neq p, \forall j>i, \ldots, N: \\
& r_{i j j}^{p}=\frac{\omega_{i}^{2}\left(\omega_{i}^{2}-2 \omega_{j}^{2}-2 \omega_{p}^{2}\right)+\left(\omega_{p}^{2}-4 \omega_{j}^{2}\right)\left(\omega_{p}^{2}-2 \omega_{j}^{2}\right)}{\left(\omega_{i}^{2}-\omega_{p}^{2}\right) D_{i j p}^{(1)}}\left[A_{i j j}^{p}+A_{j i j}^{p}+h_{i j j}^{p}\right] \\
& -\frac{2 \omega_{j}^{4}\left(\omega_{p}^{2}-4 \omega_{j}^{2}+3 \omega_{i}^{2}\right)}{\left(\omega_{i}^{2}-\omega_{p}^{2}\right) D_{i j p}^{(1)}} B_{i j j}^{p}+\frac{2 \omega_{i}^{2} \omega_{j}^{2}}{D_{i j p}^{(1)}} B_{j i j}^{p} \text {, } \\
& u_{i j j}^{p}=\frac{\omega_{i}^{2}\left(\omega_{i}^{2}-2 \omega_{j}^{2}-2 \omega_{p}^{2}\right)+\left(\omega_{p}^{2}-4 \omega_{j}^{2}\right)\left(\omega_{p}^{2}-2 \omega_{j}^{2}\right)}{\left(\omega_{i}^{2}-\omega_{p}^{2}\right) D_{i j p}^{(1)}} B_{i j j}^{p} \\
& +\frac{8 \omega_{j}^{2}-6 \omega_{i}^{2}-2 \omega_{p}^{2}}{\left(\omega_{i}^{2}-\omega_{p}^{2}\right) D_{i j p}^{(1)}}\left[A_{i j j}^{p}+A_{j i j}^{p}+h_{i j j}\right]-\frac{2 \omega_{i}^{2}}{D_{i j p}^{(1)}} B_{j i j}^{p}, \\
& u_{j i j}^{p}=\frac{1}{D_{i j p}^{(1)}}\left[4\left(A_{j i j}^{p}+A_{i j j}^{p}+h_{i j j}^{p}\right)-4 \omega_{j}^{2} B_{i j j}^{p}+\left(4 \omega_{j}^{2}-\omega_{p}^{2}+\omega_{i}^{2}\right) B_{j i j}^{p}\right], \\
& v_{i j j}^{p}=\frac{6 \omega_{i}^{2} \omega_{j}^{2}+2 \omega_{j}^{2} \omega_{p}^{2}+2 \omega_{p}^{2} \omega_{i}^{2}-8 \omega_{j}^{4}-\omega_{p}^{4}-\omega_{i}^{4}}{\left(\omega_{p}^{2}-\omega_{i}^{2}\right) D_{i j p}^{(1)}}\left[A_{i j j}^{p}+A_{j i j}^{p}+h_{i j j}^{p}\right] \\
& +\frac{2 \omega_{j}^{4}\left(3 \omega_{p}^{2}+\omega_{i}^{2}-4 \omega_{j}^{2}\right)}{\left(\omega_{p}^{2}-\omega_{i}^{2}\right) D_{i j p}^{(1)}} B_{i j j}^{p}-\frac{\omega_{j}^{2}\left(-\omega_{p}^{2}+4 \omega_{j}^{2}-\omega_{i}^{2}\right)}{D_{i j p}^{(1)}} B_{j i j}^{p}, \\
& v_{j i j}^{p}=\frac{8 \omega_{j}^{2}-2 \omega_{i}^{2}-2 \omega_{p}^{2}}{D_{i j p}^{(1)}}\left[A_{i j j}^{p}+A_{j i j}^{p}+h_{i j j}^{p}\right] \\
& +\frac{2 \omega_{j}^{2} \omega_{p}^{2}-8 \omega_{j}^{4}+2 \omega_{j}^{2} \omega_{i}^{2}}{D_{i j p}^{(1)}} B_{i j j}^{p}+\frac{\omega_{p}^{2} \omega_{i}^{2}+4 \omega_{j}^{2} \omega_{i}^{2}-\omega_{i}^{4}}{D_{i j p}^{(1)}} B_{j i j}^{p}, \\
& \mu_{i j j}^{p}=\frac{6 \omega_{i}^{2} \omega_{j}^{2}+2 \omega_{j}^{2} \omega_{p}^{2}+2 \omega_{p}^{2} \omega_{i}^{2}-8 \omega_{j}^{4}-\omega_{p}^{4}-\omega_{i}^{4}}{\left(\omega_{p}^{2}-\omega_{i}^{2}\right) D_{i j p}^{(1)}} B_{i j j}^{p} \\
& +\frac{6 \omega_{p}^{2}+2 \omega_{i}^{2}-8 \omega_{j}^{2}}{\left(\omega_{p}^{2}-\omega_{i}^{2}\right) D_{i j p}^{(1)}}\left[A_{i j j}^{p}+A_{j i j}^{p}+h_{i j j}^{p}\right]+\frac{4 \omega_{j}^{2}-\omega_{i}^{2}-\omega_{p}^{2}}{D_{i j p}^{(1)}} B_{j i j}^{p},
\end{aligned}
$$


where $D_{i j p}^{(1)}=\left(\omega_{p}+\omega_{i}-2 \omega_{j}\right)\left(\omega_{p}+\omega_{i}+2 \omega_{j}\right)\left(-\omega_{p}+\omega_{i}+2 \omega_{j}\right)\left(-\omega_{p}+\omega_{i}-2 \omega_{j}\right)$.

$$
\begin{aligned}
& \forall i=1, \ldots, N-1, \forall j>i, \ldots, N, j \neq p: \\
& r_{i i j}^{p}=\frac{2 \omega_{i}^{2}\left(4 \omega_{i}^{2}-3 \omega_{p}^{2}-\omega_{j}^{2}\right)+\left(\omega_{p}-\omega_{j}\right)^{2}\left(\omega_{p}+\omega_{j}\right)^{2}}{\left(\omega_{p}^{2}-\omega_{j}^{2}\right) D_{i j p}^{(2)}}\left[-A_{i i j}^{p}-A_{j i i}^{p}-h_{i i j}^{p}\right] \\
& -\frac{2 \omega_{i}^{4}\left(4 \omega_{i}^{2}-\omega_{p}^{2}-3 \omega_{j}^{2}\right)}{\left(\omega_{p}^{2}-\omega_{j}^{2}\right) D_{i j p}^{(2)}} B_{j i i}^{p}-\frac{2 \omega_{i}^{2} \omega_{j}^{2}}{D_{i j p}^{(2)}} B_{i i j}^{p} \\
& u_{j i i}^{p}=-\frac{2 \omega_{i}^{2}\left(4 \omega_{i}^{2}-3 \omega_{p}^{2}-\omega_{j}^{2}\right)+\left(\omega_{p}-\omega_{j}\right)^{2}\left(\omega_{p}+\omega_{j}\right)^{2}}{\left(\omega_{p}^{2}-\omega_{j}^{2}\right) D_{i j p}^{(2)}} B_{j i i}^{p} \\
& +\frac{8 \omega_{i}^{2}-2 \omega_{p}^{2}-6 \omega_{j}^{2}}{\left(\omega_{p}^{2}-\omega_{j}^{2}\right) D_{i j p}^{(2)}}\left[-A_{j i i}^{p}-A_{i i j}^{p}-h_{i i j}^{p}\right]-\frac{2 \omega_{j}^{2}}{D_{i j p}^{(2)}} B_{i i j}^{p} \text {, } \\
& u_{i i j}^{p}=\frac{1}{D_{i j p}^{(2)}}\left[4\left(A_{j i i}^{p}+A_{i i j}^{p}+h_{i i j}^{p}\right)-4 \omega_{i}^{2} B_{j i i}^{p}-\left(\omega_{p}^{2}-\omega_{j}^{2}-4 \omega_{i}^{2}\right) B_{i i j}^{p}\right], \\
& v_{j i i}^{p}=\frac{8 \omega_{i}^{4}+\omega_{j}^{4}+\omega_{p}^{4}-2 \omega_{p}^{2} \omega_{i}^{2}-6 \omega_{i}^{2} \omega_{j}^{2}-2 \omega_{j}^{2} \omega_{p}^{2}}{\left(\omega_{j}^{2}-\omega_{p}^{2}\right) D_{i j p}^{(2)}}\left[A_{j i i}^{p}+A_{i i j}^{p}+h_{i i j}^{p}\right] \\
& +\frac{8 \omega_{i}^{6}-2 \omega_{j}^{2} \omega_{i}^{4}-6 \omega_{p}^{2} \omega_{i}^{4}}{\left(\omega_{j}^{2}-\omega_{p}^{2}\right) D_{i j p}^{(2)}} B_{j i i}^{p}+\frac{\omega_{i}^{2}\left(\omega_{j}^{2}-4 \omega_{i}^{2}+\omega_{p}^{2}\right)}{D_{i j p}^{(2)}} B_{i i j}^{p}, \\
& v_{i i j}^{p}=\frac{8 \omega_{i}^{2}-2 \omega_{p}^{2}-2 \omega_{j}^{2}}{D_{i j p}^{(2)}}\left[A_{j i i}^{p}+A_{i i j}^{p}+h_{i i j}^{p}\right] \\
& +\frac{2 \omega_{i}^{2} \omega_{j}^{2}-8 \omega_{i}^{4}+2 \omega_{i}^{2} \omega_{p}^{2}}{D_{i j p}^{(2)}} B_{j i i}^{p}+\frac{-\omega_{j}^{4}+4 \omega_{i}^{2} \omega_{j}^{2}+\omega_{j}^{2} \omega_{p}^{2}}{D_{i j p}^{(2)}} B_{i j j}^{p}, \\
& \mu_{i i j}^{p}=\frac{8 \omega_{i}^{2}-2 \omega_{j}^{2}-6 \omega_{p}^{2}}{\left(\omega_{j}^{2}-\omega_{p}^{2}\right) D_{i j p}^{(2)}}\left[A_{i i j}^{p}+A_{j i i}^{p}+h_{i i j}^{p}\right]+\frac{4 \omega_{i}^{2}-\omega_{j}^{2}-\omega_{p}^{2}}{D_{i j p}^{(2)}} B_{i i j}^{p} \\
& -\frac{6 \omega_{i}^{2} \omega_{j}^{2}+2 \omega_{j}^{2} \omega_{p}^{2}+2 \omega_{i}^{2} \omega_{p}^{2}-8 \omega_{i}^{4}-\omega_{p}^{4}-\omega_{j}^{4}}{\left(\omega_{j}^{2}-\omega_{p}^{2}\right) D_{i j p}^{(2)}} B_{j i i}^{p},
\end{aligned}
$$

where $D_{i j p}^{(2)}=\left(\omega_{p}+2 \omega_{i}-\omega_{j}\right)\left(\omega_{p}+2 \omega_{i}+\omega_{j}\right)\left(-\omega_{p}+2 \omega_{i}+\omega_{j}\right)\left(-\omega_{p}+2 \omega_{i}-\omega_{j}\right)$.

$$
\forall i=1, \ldots, N-2, \forall j>i, \ldots, N-1, \forall k>j, \ldots, N \text { : }
$$




$$
\begin{aligned}
& u_{i j k}^{p}=\frac{1}{D_{i j k p}^{(3)}}\left[\left(2 \omega_{j}^{4}-6 \omega_{i}^{4}-4 \omega_{k}^{2} \omega_{p}^{2}+4 \omega_{i}^{2} \omega_{p}^{2}+4 \omega_{i}^{2} \omega_{k}^{2}+2 \omega_{k}^{4}\right.\right. \\
& \left.+4 \omega_{i}^{2} \omega_{j}^{2}+2 \omega_{p}^{4}-4 \omega_{k}^{2} \omega_{j}^{2}-4 \omega_{j}^{2} \omega_{p}^{2}\right)\left(h_{i j k}^{p}+A_{i j k}^{p}+A_{k i j}^{p}+A_{j i k}^{p}\right) \\
& +\left(-\omega_{k}^{2} \omega_{j}^{4}-\omega_{i}^{2} \omega_{k}^{4}-2 \omega_{j}^{2} \omega_{i}^{2} \omega_{p}^{2}+3 \omega_{j}^{2} \omega_{p}^{4}-\omega_{k}^{4} \omega_{j}^{2}-2 \omega_{i}^{2} \omega_{k}^{2} \omega_{p}^{2}\right. \\
& +3 \omega_{i}^{2} \omega_{p}^{4}-\omega_{j}^{4} \omega_{i}^{2}-\omega_{i}^{4} \omega_{k}^{2}+3 \omega_{k}^{2} \omega_{p}^{4}+\omega_{i}^{6}-\omega_{p}^{6}-3 \omega_{k}^{4} \omega_{p}^{2}+\omega_{k}^{6}-3 \omega_{j}^{4} \omega_{p}^{2} \\
& \left.+10 \omega_{i}^{2} \omega_{k}^{2} \omega_{j}^{2}+\omega_{j}^{6}-2 \omega_{k}^{2} \omega_{j}^{2} \omega_{p}^{2}-3 \omega_{i}^{4} \omega_{p}^{2}-\omega_{j}^{2} \omega_{i}^{4}\right) B_{i j k}^{p} \\
& +\left(-2 \omega_{j}^{4} \omega_{i}^{2}+4 \omega_{i}^{4} \omega_{p}^{2}+4 \omega_{j}^{2} \omega_{i}^{4}+6 \omega_{i}^{2} \omega_{k}^{4}-4 \omega_{i}^{4} \omega_{k}^{2}\right. \\
& \left.+4 \omega_{j}^{2} \omega_{i}^{2} \omega_{p}^{2}-2 \omega_{i}^{2} \omega_{p}^{4}-2 \omega_{i}^{6}-4 \omega_{i}^{2} \omega_{k}^{2} \omega_{p}^{2}-4 \omega_{i}^{2} \omega_{k}^{2} \omega_{j}^{2}\right) B_{j i k}^{p} \\
& +\left(-2 \omega_{i}^{2} \omega_{p}^{4}+6 \omega_{j}^{4} \omega_{i}^{2}+4 \omega_{i}^{2} \omega_{k}^{2} \omega_{p}^{2}-4 \omega_{i}^{2} \omega_{k}^{2} \omega_{j}^{2}-4 \omega_{j}^{2} \omega_{i}^{2} \omega_{p}^{2}\right. \\
& \left.\left.-4 \omega_{j}^{2} \omega_{i}^{4}-2 \omega_{i}^{2} \omega_{k}^{4}+4 \omega_{i}^{4} \omega_{p}^{2}-2 \omega_{i}^{6}+4 \omega_{i}^{4} \omega_{k}^{2}\right) B_{k i j}^{p}\right] \text {, } \\
& u_{j i k}^{p}=\frac{1}{D_{i j k p}^{(3)}}\left[\left(-2 \omega_{p}^{4}+6 \omega_{j}^{4}-2 \omega_{i}^{4}-4 \omega_{k}^{2} \omega_{j}^{2}+4 \omega_{k}^{2} \omega_{p}^{2}\right.\right. \\
& \left.-2 \omega_{k}^{4}+4 \omega_{i}^{2} \omega_{k}^{2}-4 \omega_{i}^{2} \omega_{j}^{2}+4 \omega_{i}^{2} \omega_{p}^{2}-4 \omega_{j}^{2} \omega_{p}^{2}\right)\left(h_{i j k}^{p}+A_{i j k}^{p}+A_{k i j}^{p}+A_{j i k}^{p}\right) \\
& +\left(2 \omega_{j}^{6}+4 \omega_{k}^{2} \omega_{j}^{2} \omega_{p}^{2}+4 \omega_{i}^{2} \omega_{k}^{2} \omega_{j}^{2}+2 \omega_{j}^{2} \omega_{i}^{4}+4 \omega_{k}^{2} \omega_{j}^{4}\right. \\
& \left.-4 \omega_{j}^{2} \omega_{i}^{2} \omega_{p}^{2}+2 \omega_{j}^{2} \omega_{p}^{4}-4 \omega_{j}^{4} \omega_{i}^{2}-4 \omega_{j}^{4} \omega_{p}^{2}-6 \omega_{k}^{4} \omega_{j}^{2}\right) B_{i j k}^{p} \\
& +\left(3 \omega_{j}^{4} \omega_{p}^{2}+\omega_{k}^{2} \omega_{j}^{4}-3 \omega_{k}^{2} \omega_{p}^{4}+\omega_{i}^{2} \omega_{k}^{4}+\omega_{k}^{4} \omega_{j}^{2}+3 \omega_{i}^{4} \omega_{p}^{2}\right. \\
& +2 \omega_{i}^{2} \omega_{k}^{2} \omega_{p}^{2}+\omega_{j}^{4} \omega_{i}^{2}+\omega_{i}^{4} \omega_{k}^{2}-\omega_{i}^{6}+3 \omega_{k}^{4} \omega_{p}^{2}-\omega_{j}^{6}-\omega_{k}^{6}+2 \omega_{j}^{2} \omega_{i}^{2} \omega_{p}^{2}+\omega_{p}^{6} \\
& \left.+2 \omega_{k}^{2} \omega_{j}^{2} \omega_{p}^{2}-3 \omega_{j}^{2} \omega_{p}^{4}-10 \omega_{i}^{2} \omega_{k}^{2} \omega_{j}^{2}-3 \omega_{i}^{2} \omega_{p}^{4}+\omega_{j}^{2} \omega_{i}^{4}\right) B_{j i k}^{p} \\
& +\left(4 \omega_{i}^{2} \omega_{k}^{2} \omega_{j}^{2}+4 \omega_{j}^{4} \omega_{i}^{2}+2 \omega_{j}^{2} \omega_{p}^{4}-6 \omega_{j}^{2} \omega_{i}^{4}+2 \omega_{k}^{4} \omega_{j}^{2}-4 \omega_{j}^{4} \omega_{p}^{2}\right. \\
& \left.\left.+2 \omega_{j}^{6}-4 \omega_{k}^{2} \omega_{j}^{4}-4 \omega_{k}^{2} \omega_{j}^{2} \omega_{p}^{2}+4 \omega_{j}^{2} \omega_{i}^{2} \omega_{p}^{2}\right) B_{k i j}^{p}\right] \text {, } \\
& u_{k i j}^{p}=\frac{1}{D_{i j k p}^{(3)}}\left[\left(2 \omega_{p}^{4}+2 \omega_{j}^{4}+4 \omega_{i}^{2} \omega_{k}^{2}-6 \omega_{k}^{4}+4 \omega_{k}^{2} \omega_{p}^{2}-4 \omega_{i}^{2} \omega_{p}^{2}\right.\right. \\
& \left.+4 \omega_{k}^{2} \omega_{j}^{2}-4 \omega_{i}^{2} \omega_{j}^{2}-4 \omega_{j}^{2} \omega_{p}^{2}+2 \omega_{i}^{4}\right)\left(h_{i j k}^{p}+A_{i j k}^{p}+A_{k i j}^{p}+A_{j i k}^{p}\right) \\
& +\left(-4 \omega_{i}^{2} \omega_{k}^{2} \omega_{j}^{2}-2 \omega_{k}^{6}-2 \omega_{i}^{4} \omega_{k}^{2}-4 \omega_{k}^{2} \omega_{j}^{2} \omega_{p}^{2}+4 \omega_{i}^{2} \omega_{k}^{2} \omega_{p}^{2}\right. \\
& \left.+6 \omega_{k}^{2} \omega_{j}^{4}-2 \omega_{k}^{2} \omega_{p}^{4}+4 \omega_{i}^{2} \omega_{k}^{4}-4 \omega_{k}^{4} \omega_{j}^{2}+4 \omega_{k}^{4} \omega_{p}^{2}\right) B_{i j k}^{p} \\
& +\left(-2 \omega_{k}^{6}-4 \omega_{i}^{2} \omega_{k}^{4}+4 \omega_{k}^{2} \omega_{j}^{2} \omega_{p}^{2}-4 \omega_{i}^{2} \omega_{k}^{2} \omega_{p}^{2}-2 \omega_{k}^{2} \omega_{p}^{4}+6 \omega_{i}^{4} \omega_{k}^{2}\right. \\
& \left.-4 \omega_{i}^{2} \omega_{k}^{2} \omega_{j}^{2}+4 \omega_{k}^{4} \omega_{j}^{2}-2 \omega_{k}^{2} \omega_{j}^{4}+4 \omega_{k}^{4} \omega_{p}^{2}\right) B_{j i k}^{p} \\
& +\left(-\omega_{k}^{2} \omega_{j}^{4}-\omega_{i}^{2} \omega_{k}^{4}-2 \omega_{j}^{2} \omega_{i}^{2} \omega_{p}^{2}+3 \omega_{j}^{2} \omega_{p}^{4}-\omega_{k}^{4} \omega_{j}^{2}-2 \omega_{i}^{2} \omega_{k}^{2} \omega_{p}^{2}\right. \\
& +3 \omega_{i}^{2} \omega_{p}^{4}-\omega_{j}^{4} \omega_{i}^{2}-\omega_{i}^{4} \omega_{k}^{2}+3 \omega_{k}^{2} \omega_{p}^{4}+\omega_{i}^{6}-\omega_{p}^{6}-3 \omega_{k}^{4} \omega_{p}^{2}+\omega_{k}^{6} \\
& \left.\left.-3 \omega_{j}^{4} \omega_{p}^{2}+10 \omega_{i}^{2} \omega_{k}^{2} \omega_{j}^{2}+\omega_{j}^{6}-2 \omega_{k}^{2} \omega_{j}^{2} \omega_{p}^{2}-3 \omega_{i}^{4} \omega_{p}^{2}-\omega_{j}^{2} \omega_{i}^{4}\right) B_{k i j}^{p}\right] \text {, }
\end{aligned}
$$




$$
\begin{aligned}
r_{i j k}^{p}= & \frac{1}{D_{i j k p}^{(3)}}\left[\left(-\omega_{k}^{2} \omega_{j}^{4}-\omega_{i}^{2} \omega_{k}^{4}-2 \omega_{j}^{2} \omega_{i}^{2} \omega_{p}^{2}+3 \omega_{j}^{2} \omega_{p}^{4}-\omega_{k}^{4} \omega_{j}^{2}\right.\right. \\
& -2 \omega_{i}^{2} \omega_{k}^{2} \omega_{p}^{2}+3 \omega_{i}^{2} \omega_{p}^{4}-\omega_{j}^{4} \omega_{i}^{2}-\omega_{i}^{4} \omega_{k}^{2}+3 \omega_{k}^{2} \omega_{p}^{4}+\omega_{i}^{6}-\omega_{p}^{6}-3 \omega_{k}^{4} \omega_{p}^{2}+\omega_{k}^{6} \\
& \left.-3 \omega_{j}^{4} \omega_{p}^{2}+10 \omega_{i}^{2} \omega_{k}^{2} \omega_{j}^{2}+\omega_{j}^{6}-2 \omega_{k}^{2} \omega_{j}^{2} \omega_{p}^{2}-3 \omega_{i}^{4} \omega_{p}^{2}-\omega_{j}^{2} \omega_{i}^{4}\right)\left(h_{i j k}^{p}+A_{i j k}^{p}+A_{k i j}^{p}+A_{j i k}^{p}\right) \\
& +\left(4 \omega_{i}^{2} \omega_{k}^{2} \omega_{j}^{4}-4 \omega_{k}^{2} \omega_{j}^{4} \omega_{p}^{2}+4 \omega_{i}^{2} \omega_{k}^{4} \omega_{j}^{2}-4 \omega_{j}^{2} \omega_{k}^{4} \omega_{p}^{2}-6 \omega_{i}^{4} \omega_{k}^{2} \omega_{j}^{2}+2 \omega_{k}^{2} \omega_{j}^{2} \omega_{p}^{4}\right. \\
& \left.+4 \omega_{i}^{2} \omega_{k}^{2} \omega_{j}^{2} \omega_{p}^{2}-4 \omega_{j}^{4} \omega_{k}^{4}+2 \omega_{k}^{2} \omega_{j}^{6}+2 \omega_{j}^{2} \omega_{k}^{6}\right) B_{i j k}^{p} \\
& +\left(4 \omega_{i}^{2} \omega_{k}^{4} \omega_{j}^{2}+4 \omega_{i}^{2} \omega_{k}^{2} \omega_{j}^{2} \omega_{p}^{2}+2 \omega_{i}^{2} \omega_{k}^{2} \omega_{p}^{4}+2 \omega_{i}^{2} \omega_{k}^{6}+2 \omega_{i}^{6} \omega_{k}^{2}-4 \omega_{i}^{4} \omega_{k}^{4}\right. \\
& \left.-6 \omega_{i}^{2} \omega_{k}^{2} \omega_{j}^{4}-4 \omega_{i}^{2} \omega_{k}^{4} \omega_{p}^{2}-4 \omega_{i}^{4} \omega_{k}^{2} \omega_{p}^{2}+4 \omega_{i}^{4} \omega_{k}^{2} \omega_{j}^{2}\right) B_{j i k}^{p} \\
& +\left(-4 \omega_{i}^{4} \omega_{j}^{4}+2 \omega_{i}^{2} \omega_{j}^{2} \omega_{p}^{4}-6 \omega_{i}^{2} \omega_{k}^{4} \omega_{j}^{2}+2 \omega_{i}^{2} \omega_{j}^{6}+2 \omega_{i}^{6} \omega_{j}^{2}-4 \omega_{i}^{2} \omega_{j}^{4} \omega_{p}^{2}\right. \\
& \left.\left.+4 \omega_{i}^{2} \omega_{k}^{2} \omega_{j}^{2} \omega_{p}^{2}+4 \omega_{i}^{2} \omega_{k}^{2} \omega_{j}^{4}-4 \omega_{i}^{4} \omega_{j}^{2} \omega_{p}^{2}+4 \omega_{i}^{4} \omega_{k}^{2} \omega_{j}^{2}\right) B_{k i j}^{p}\right]
\end{aligned}
$$$$
\begin{aligned}
\mu_{i j k}^{p}= & \frac{1}{D_{i j k p}^{(3)}}\left[\left(-4 \omega_{i}^{2} \omega_{j}^{2}+4 \omega_{i}^{2} \omega_{p}^{2}+12 \omega_{i}^{2} \omega_{k}^{2}-4 \omega_{k}^{2} \omega_{j}^{2}+4 \omega_{k}^{2} \omega_{p}^{2}+2 \omega_{p}^{4}\right.\right. \\
& \left.+10 \omega_{j}^{4}-6 \omega_{i}^{4}-6 \omega_{k}^{4}-12 \omega_{j}^{2} \omega_{p}^{2}\right)\left(h_{i j k}^{p}+A_{i j k}^{p}+A_{k i j}^{p}+A_{j i k}^{p}\right) \\
& +\left(10 \omega_{i}^{2} \omega_{k}^{2} \omega_{j}^{2}-2 \omega_{k}^{2} \omega_{j}^{2} \omega_{p}^{2}+2 \omega_{i}^{2} \omega_{k}^{2} \omega_{p}^{2}-6 \omega_{j}^{2} \omega_{i}^{2} \omega_{p}^{2}+9 \omega_{k}^{2} \omega_{j}^{4}\right. \\
& +3 \omega_{j}^{6}-11 \omega_{k}^{4} \omega_{j}^{2}+\omega_{k}^{2} \omega_{p}^{4}+3 \omega_{i}^{2} \omega_{k}^{4}+\omega_{k}^{4} \omega_{p}^{2}-\omega_{k}^{6}-3 \omega_{i}^{4} \omega_{p}^{2} \\
& \left.+3 \omega_{i}^{2} \omega_{p}^{4}+\omega_{i}^{6}-\omega_{p}^{6}+5 \omega_{j}^{2} \omega_{p}^{4}-7 \omega_{j}^{4} \omega_{p}^{2}-5 \omega_{j}^{4} \omega_{i}^{2}+\omega_{j}^{2} \omega_{i}^{4}-3 \omega_{i}^{4} \omega_{k}^{2}\right) B_{i j k}^{p} \\
& +\left(-18 \omega_{i}^{2} \omega_{k}^{2} \omega_{j}^{2}-3 \omega_{j}^{2} \omega_{p}^{4}+5 \omega_{k}^{4} \omega_{j}^{2}+5 \omega_{j}^{2} \omega_{i}^{4}-\omega_{k}^{2} \omega_{j}^{4}-\omega_{j}^{4} \omega_{i}^{2}+7 \omega_{i}^{4} \omega_{p}^{2}\right. \\
& +3 \omega_{i}^{2} \omega_{k}^{4}+\omega_{p}^{6}-\omega_{j}^{6}-3 \omega_{k}^{6}-3 \omega_{i}^{6}+3 \omega_{j}^{4} \omega_{p}^{2}+7 \omega_{k}^{4} \omega_{p}^{2}+3 \omega_{i}^{4} \omega_{k}^{2}+6 \omega_{k}^{2} \omega_{j}^{2} \omega_{p}^{2} \\
& \left.+6 \omega_{j}^{2} \omega_{i}^{2} \omega_{p}^{2}-5 \omega_{k}^{2} \omega_{p}^{4}-6 \omega_{i}^{2} \omega_{k}^{2} \omega_{p}^{2}-5 \omega_{i}^{2} \omega_{p}^{4}\right) B_{j i k}^{p} \\
& +\left(\omega_{k}^{4} \omega_{j}^{2}+\omega_{k}^{6}-2 \omega_{j}^{2} \omega_{i}^{2} \omega_{p}^{2}+10 \omega_{i}^{2} \omega_{k}^{2} \omega_{j}^{2}+3 \omega_{k}^{2} \omega_{p}^{4}-6 \omega_{k}^{2} \omega_{j}^{2} \omega_{p}^{2}-3 \omega_{i}^{2} \omega_{k}^{4}\right. \\
& +2 \omega_{i}^{2} \omega_{k}^{2} \omega_{p}^{2}+5 \omega_{j}^{2} \omega_{p}^{4}+3 \omega_{i}^{4} \omega_{k}^{2}-11 \omega_{j}^{2} \omega_{i}^{4}-3 \omega_{k}^{4} \omega_{p}^{2}+\omega_{i}^{4} \omega_{p}^{2}+3 \omega_{j}^{6} \\
& \left.\left.+\omega_{i}^{2} \omega_{p}^{4}-\omega_{i}^{6}-\omega_{p}^{6}+9 \omega_{j}^{4} \omega_{i}^{2}-7 \omega_{j}^{4} \omega_{p}^{2}-5 \omega_{k}^{2} \omega_{j}^{4}\right) B_{k i j}^{p}\right]
\end{aligned}
$$$$
v_{k i j}^{p}=\frac{1}{D_{i j k p}^{(3)}}\left[\left(-2 \omega_{j}^{2} \omega_{i}^{2} \omega_{p}^{2}+\omega_{j}^{4} \omega_{p}^{2}-\omega_{j}^{6}+3 \omega_{k}^{2} \omega_{j}^{4}+\omega_{k}^{6}-7 \omega_{i}^{4} \omega_{p}^{2}-11 \omega_{j}^{4} \omega_{i}^{2}+2 \omega_{k}^{2} \omega_{j}^{2} \omega_{p}^{2}\right.\right.
$$$$
+9 \omega_{j}^{2} \omega_{i}^{4}+3 \omega_{i}^{6}+5 \omega_{i}^{2} \omega_{p}^{4}+\omega_{j}^{2} \omega_{p}^{4}-5 \omega_{i}^{4} \omega_{k}^{2}+10 \omega_{i}^{2} \omega_{k}^{2} \omega_{j}^{2}-6 \omega_{i}^{2} \omega_{k}^{2} \omega_{p}^{2}+3 \omega_{k}^{2} \omega_{p}^{4}
$$$$
\left.-3 \omega_{k}^{4} \omega_{p}^{2}-\omega_{p}^{6}+\omega_{i}^{2} \omega_{k}^{4}-3 \omega_{k}^{4} \omega_{j}^{2}\right)\left(h_{i j k}^{p}+A_{i j k}^{p}+A_{k i j}^{p}+A_{j i k}^{p}\right)
$$$$
+\left(3 \omega_{i}^{4} \omega_{p}^{4}+\omega_{i}^{8}-6 \omega_{i}^{4} \omega_{k}^{2} \omega_{p}^{2}-5 \omega_{i}^{4} \omega_{k}^{4}+6 \omega_{i}^{2} \omega_{k}^{2} \omega_{j}^{2} \omega_{p}^{2}+5 \omega_{i}^{2} \omega_{j}^{2} \omega_{p}^{4}-7 \omega_{i}^{2} \omega_{j}^{4} \omega_{p}^{2}\right.
$$$$
+18 \omega_{i}^{4} \omega_{k}^{2} \omega_{j}^{2}+3 \omega_{i}^{2} \omega_{k}^{6}-3 \omega_{i}^{2} \omega_{k}^{2} \omega_{j}^{4}-7 \omega_{i}^{2} \omega_{k}^{4} \omega_{p}^{2}-6 \omega_{i}^{4} \omega_{j}^{2} \omega_{p}^{2}+\omega_{i}^{6} \omega_{k}^{2}-3 \omega_{i}^{2} \omega_{k}^{4} \omega_{j}^{2}
$$$$
\left.+5 \omega_{i}^{2} \omega_{k}^{2} \omega_{p}^{4}+\omega_{i}^{6} \omega_{j}^{2}-5 \omega_{i}^{4} \omega_{j}^{4}-3 \omega_{i}^{6} \omega_{p}^{2}-\omega_{i}^{2} \omega_{p}^{6}+3 \omega_{i}^{2} \omega_{j}^{6}\right) B_{j i k}^{p}
$$ 


$$
\begin{aligned}
& +\left(3 \omega_{k}^{2} \omega_{j}^{6}+6 \omega_{i}^{2} \omega_{j}^{4} \omega_{p}^{2}+5 \omega_{i}^{4} \omega_{j}^{4}+7 \omega_{i}^{4} \omega_{j}^{2} \omega_{p}^{2}-9 \omega_{i}^{4} \omega_{k}^{2} \omega_{j}^{2}-\omega_{i}^{2} \omega_{j}^{6}+\omega_{p}^{6} \omega_{j}^{2}-\omega_{j}^{2} \omega_{k}^{4} \omega_{p}^{2}\right. \\
& -3 \omega_{p}^{4} \omega_{j}^{4}+3 \omega_{j}^{6} \omega_{p}^{2}+11 \omega_{i}^{2} \omega_{k}^{4} \omega_{j}^{2}-3 \omega_{i}^{6} \omega_{j}^{2}-5 \omega_{i}^{2} \omega_{j}^{2} \omega_{p}^{4}-2 \omega_{k}^{2} \omega_{j}^{4} \omega_{p}^{2}-\omega_{k}^{2} \omega_{j}^{2} \omega_{p}^{4} \\
& \left.+2 \omega_{i}^{2} \omega_{k}^{2} \omega_{j}^{2} \omega_{p}^{2}+\omega_{j}^{2} \omega_{k}^{6}-3 \omega_{j}^{4} \omega_{k}^{4}-10 \omega_{i}^{2} \omega_{k}^{2} \omega_{j}^{4}-\omega_{j}^{8}\right) B_{i j k}^{p} \\
& +\left(-4 \omega_{i}^{4} \omega_{k}^{2} \omega_{j}^{2}-6 \omega_{i}^{2} \omega_{k}^{4} \omega_{j}^{2}-4 \omega_{i}^{4} \omega_{j}^{4}-12 \omega_{i}^{4} \omega_{j}^{2} \omega_{p}^{2}+2 \omega_{i}^{2} \omega_{j}^{2} \omega_{p}^{4}+4 \omega_{i}^{2} \omega_{j}^{4} \omega_{p}^{2}\right. \\
& \left.\left.+12 \omega_{i}^{2} \omega_{k}^{2} \omega_{j}^{4}+10 \omega_{i}^{6} \omega_{j}^{2}-6 \omega_{i}^{2} \omega_{j}^{6}+4 \omega_{i}^{2} \omega_{k}^{2} \omega_{j}^{2} \omega_{p}^{2}\right) B_{k i j}^{p}\right] \text {, } \\
& v_{j i k}^{p}=\frac{1}{D_{i j k p}^{(3)}}\left[\left(\omega_{i}^{2} \omega_{k}^{4}+\omega_{i}^{4} \omega_{p}^{2}+3 \omega_{j}^{2} \omega_{i}^{4}+\omega_{k}^{4} \omega_{p}^{2}-\omega_{p}^{6}-3 \omega_{j}^{4} \omega_{p}^{2}+3 \omega_{j}^{2} \omega_{p}^{4}+2 \omega_{j}^{2} \omega_{i}^{2} \omega_{p}^{2}+\omega_{j}^{6}\right.\right. \\
& +3 \omega_{k}^{4} \omega_{j}^{2}+\omega_{i}^{2} \omega_{p}^{4}+2 \omega_{k}^{2} \omega_{j}^{2} \omega_{p}^{2}-\omega_{k}^{6}+2 \omega_{i}^{2} \omega_{k}^{2} \omega_{j}^{2}-10 \omega_{i}^{2} \omega_{k}^{2} \omega_{p}^{2}-3 \omega_{j}^{4} \omega_{i}^{2} \\
& \left.+\omega_{i}^{4} \omega_{k}^{2}-3 \omega_{k}^{2} \omega_{j}^{4}-\omega_{i}^{6}+\omega_{k}^{2} \omega_{p}^{4}\right)\left(h_{i j k}^{p}+A_{i j k}^{p}+A_{k i j}^{p}+A_{j i k}^{p}\right) \\
& +\left(-3 \omega_{i}^{4} \omega_{k}^{4}-\omega_{i}^{2} \omega_{k}^{2} \omega_{p}^{4}-2 \omega_{i}^{2} \omega_{k}^{4} \omega_{j}^{2}-2 \omega_{i}^{2} \omega_{k}^{4} \omega_{p}^{2}-\omega_{i}^{4} \omega_{k}^{2} \omega_{p}^{2}+\omega_{i}^{6} \omega_{k}^{2}\right. \\
& +\omega_{k}^{2} \omega_{j}^{6}-2 \omega_{j}^{2} \omega_{k}^{4} \omega_{p}^{2}-\omega_{i}^{4} \omega_{k}^{2} \omega_{j}^{2}+3 \omega_{i}^{2} \omega_{k}^{6}-\omega_{k}^{2} \omega_{j}^{2} \omega_{p}^{4}-3 \omega_{p}^{4} \omega_{k}^{4}+3 \omega_{k}^{6} \omega_{p}^{2}-\omega_{k}^{2} \omega_{j}^{4} \omega_{p}^{2} \\
& \left.-\omega_{k}^{8}+10 \omega_{i}^{2} \omega_{k}^{2} \omega_{j}^{2} \omega_{p}^{2}+3 \omega_{j}^{2} \omega_{k}^{6}-3 \omega_{j}^{4} \omega_{k}^{4}-\omega_{i}^{2} \omega_{k}^{2} \omega_{j}^{4}+\omega_{k}^{2} \omega_{p}^{6}\right) B_{i j k}^{p} \\
& +\left(-4 \omega_{i}^{2} \omega_{k}^{2} \omega_{j}^{2} \omega_{p}^{2}+4 \omega_{i}^{2} \omega_{k}^{4} \omega_{j}^{2}+4 \omega_{i}^{4} \omega_{k}^{2} \omega_{j}^{2}-2 \omega_{i}^{2} \omega_{k}^{6}+6 \omega_{i}^{2} \omega_{k}^{2} \omega_{p}^{4}-4 \omega_{i}^{4} \omega_{k}^{2} \omega_{p}^{2}\right. \\
& \left.-2 \omega_{i}^{6} \omega_{k}^{2}+4 \omega_{i}^{4} \omega_{k}^{4}-2 \omega_{i}^{2} \omega_{k}^{2} \omega_{j}^{4}-4 \omega_{i}^{2} \omega_{k}^{4} \omega_{p}^{2}\right) B_{j i k}^{p} \\
& +\left(-2 \omega_{i}^{4} \omega_{j}^{2} \omega_{p}^{2}+3 \omega_{i}^{6} \omega_{k}^{2}-3 \omega_{i}^{4} \omega_{k}^{4}+3 \omega_{i}^{6} \omega_{p}^{2}-3 \omega_{i}^{4} \omega_{p}^{4}-\omega_{i}^{2} \omega_{j}^{2} \omega_{p}^{4}-2 \omega_{i}^{4} \omega_{k}^{2} \omega_{p}^{2}\right. \\
& -\omega_{i}^{8}-\omega_{i}^{2} \omega_{k}^{4} \omega_{p}^{2}+\omega_{i}^{2} \omega_{j}^{6}-2 \omega_{i}^{4} \omega_{k}^{2} \omega_{j}^{2}-\omega_{i}^{2} \omega_{k}^{2} \omega_{p}^{4}+3 \omega_{i}^{6} \omega_{j}^{2}+10 \omega_{i}^{2} \omega_{k}^{2} \omega_{j}^{2} \omega_{p}^{2} \\
& \left.\left.+\omega_{i}^{2} \omega_{k}^{6}+\omega_{i}^{2} \omega_{p}^{6}-\omega_{i}^{2} \omega_{k}^{2} \omega_{j}^{4}-3 \omega_{i}^{4} \omega_{j}^{4}-\omega_{i}^{2} \omega_{j}^{4} \omega_{p}^{2}-\omega_{i}^{2} \omega_{k}^{4} \omega_{j}^{2}\right) B_{k i j}^{p}\right] \text {, } \\
& v_{i j k}^{p}=\frac{1}{D_{i j k p}^{(3)}}\left[\left(-11 \omega_{k}^{2} \omega_{j}^{4}-3 \omega_{i}^{4} \omega_{p}^{2}+9 \omega_{k}^{4} \omega_{j}^{2}-\omega_{p}^{6}+\omega_{j}^{4} \omega_{p}^{2}-7 \omega_{k}^{4} \omega_{p}^{2}-\omega_{j}^{6}+\omega_{i}^{4} \omega_{k}^{2}\right.\right. \\
& +2 \omega_{j}^{2} \omega_{i}^{2} \omega_{p}^{2}-3 \omega_{j}^{2} \omega_{i}^{4}+3 \omega_{k}^{6}-6 \omega_{i}^{2} \omega_{k}^{2} \omega_{p}^{2}+\omega_{j}^{2} \omega_{p}^{4}+3 \omega_{i}^{2} \omega_{p}^{4}+5 \omega_{k}^{2} \omega_{p}^{4}-5 \omega_{i}^{2} \omega_{k}^{4} \\
& \left.+\omega_{i}^{6}+3 \omega_{j}^{4} \omega_{i}^{2}-2 \omega_{k}^{2} \omega_{j}^{2} \omega_{p}^{2}+10 \omega_{i}^{2} \omega_{k}^{2} \omega_{j}^{2}\right)\left(h_{i j k}^{p}+A_{i j k}^{p}+A_{k i j}^{p}+A_{j i k}^{p}\right) \\
& +\left(12 \omega_{i}^{2} \omega_{k}^{2} \omega_{j}^{4}-6 \omega_{k}^{2} \omega_{j}^{6}-4 \omega_{i}^{2} \omega_{k}^{4} \omega_{j}^{2}+4 \omega_{i}^{2} \omega_{k}^{2} \omega_{j}^{2} \omega_{p}^{2}+4 \omega_{k}^{2} \omega_{j}^{4} \omega_{p}^{2}\right. \\
& \left.+2 \omega_{k}^{2} \omega_{j}^{2} \omega_{p}^{4}-4 \omega_{j}^{4} \omega_{k}^{4}-12 \omega_{j}^{2} \omega_{k}^{4} \omega_{p}^{2}-6 \omega_{i}^{4} \omega_{k}^{2} \omega_{j}^{2}+10 \omega_{j}^{2} \omega_{k}^{6}\right) B_{i j k}^{p} \\
& +\left(3 \omega_{k}^{2} \omega_{j}^{6}+6 \omega_{i}^{2} \omega_{k}^{2} \omega_{j}^{2} \omega_{p}^{2}+\omega_{j}^{2} \omega_{k}^{6}-3 \omega_{i}^{2} \omega_{k}^{2} \omega_{j}^{4}-\omega_{k}^{2} \omega_{p}^{6}-3 \omega_{i}^{4} \omega_{k}^{2} \omega_{j}^{2}\right. \\
& +18 \omega_{i}^{2} \omega_{k}^{4} \omega_{j}^{2}-6 \omega_{i}^{2} \omega_{k}^{4} \omega_{p}^{2}-5 \omega_{j}^{4} \omega_{k}^{4}+3 \omega_{i}^{6} \omega_{k}^{2}+\omega_{i}^{2} \omega_{k}^{6}+5 \omega_{i}^{2} \omega_{k}^{2} \omega_{p}^{4}-6 \omega_{j}^{2} \omega_{k}^{4} \omega_{p}^{2} \\
& \left.+\omega_{k}^{8}+5 \omega_{k}^{2} \omega_{j}^{2} \omega_{p}^{4}-3 \omega_{k}^{6} \omega_{p}^{2}+3 \omega_{p}^{4} \omega_{k}^{4}-7 \omega_{i}^{4} \omega_{k}^{2} \omega_{p}^{2}-5 \omega_{i}^{4} \omega_{k}^{4}-7 \omega_{k}^{2} \omega_{j}^{4} \omega_{p}^{2}\right) B_{j i k}^{p} \\
& +\left(-\omega_{i}^{4} \omega_{j}^{2} \omega_{p}^{2}-\omega_{j}^{8}-5 \omega_{k}^{2} \omega_{j}^{2} \omega_{p}^{4}+7 \omega_{j}^{2} \omega_{k}^{4} \omega_{p}^{2}+5 \omega_{j}^{4} \omega_{k}^{4}+3 \omega_{j}^{6} \omega_{p}^{2}-\omega_{k}^{2} \omega_{j}^{6}\right. \\
& -3 \omega_{p}^{4} \omega_{j}^{4}+\omega_{i}^{6} \omega_{j}^{2}+2 \omega_{i}^{2} \omega_{k}^{2} \omega_{j}^{2} \omega_{p}^{2}+11 \omega_{i}^{4} \omega_{k}^{2} \omega_{j}^{2}+\omega_{p}^{6} \omega_{j}^{2}-10 \omega_{i}^{2} \omega_{k}^{2} \omega_{j}^{4}-3 \omega_{j}^{2} \omega_{k}^{6} \\
& \left.\left.-3 \omega_{i}^{4} \omega_{j}^{4}-9 \omega_{i}^{2} \omega_{k}^{4} \omega_{j}^{2}+3 \omega_{i}^{2} \omega_{j}^{6}+6 \omega_{k}^{2} \omega_{j}^{4} \omega_{p}^{2}-\omega_{i}^{2} \omega_{j}^{2} \omega_{p}^{4}-2 \omega_{i}^{2} \omega_{j}^{4} \omega_{p}^{2}\right) B_{k i j}^{p}\right] \text {, }
\end{aligned}
$$


where $\quad D_{i j k p}^{(3)}=\left(\omega_{k}+\omega_{i}-\omega_{p}-\omega_{j}\right)\left(\omega_{k}+\omega_{i}-\omega_{p}+\omega_{j}\right)\left(-\omega_{k}+\omega_{i}+\omega_{p}+\omega_{j}\right)\left(-\omega_{k}+\omega_{i}+\omega_{p}-\right.$ $\left.\omega_{j}\right)\left(\omega_{k}+\omega_{i}+\omega_{p}-\omega_{j}\right)\left(\omega_{k}+\omega_{i}+\omega_{p}+\omega_{j}\right)\left(-\omega_{k}+\omega_{i}-\omega_{p}+\omega_{j}\right)\left(-\omega_{k}+\omega_{i}-\omega_{p}-\omega_{j}\right)$.

\section{References}

[1] A.H. Nayfeh, D.T. Mook, Non-Linear Oscillations, Wiley, New York, 1979.

[2] R. Benamar, M.M. Bennouna, R.G. White, The effects of large vibration amplitudes on the mode shapes and natural frequencies of thin elastic structures. Part I: simply supported and clamped-clamped beams, Journal of Sound and Vibration 149 (2) (1991) 179-195.

[3] M. Amabili, F. Pellicano, M.P. Païdoussis, Nonlinear vibrations of simply supported, circular cylindrical shells, coupled to quiescent fluid, Journal of Fluids and Structures 12 (1998) 883-918.

[4] M. Amabili, Theory and experiments for large-amplitude vibrations of empty and fluid-filled circular cylindrical shells with imperfections, Journal of Sound and Vibration 262 (4) (2003) 921-975.

[5] W. Lacarbonara, A Theoretical and Experimental Investigation of Nonlinear Vibrations of Buckled Beams, Ph.D. Thesis, Virginia Polytechnic Institute and State University, 1997.

[6] A.H. Nayfeh, J.F. Nayfeh, D.T. Mook, On methods for continuous systems with quadratic and cubic nonlinearities, Nonlinear Dynamics 3 (1992) 145-162.

[7] M. Pakdemirli, S.A. Nayfeh, A.H. Nayfeh, Analysis of one-to-one autoparametric resonances in cables. Discretization vs. direct treatment, Nonlinear Dynamics 8 (1995) 65-83.

[8] A.H. Nayfeh, W. Lacarbonara, On the discretization of distributed-parameter systems with quadratic and cubic nonlinearities, Nonlinear Dynamics 13 (1997) 203-220.

[9] A.H. Nayfeh, Reduced-order models of weakly non-linear spatially continuous systems, Nonlinear Dynamics 16 (1998) 105-125.

[10] S.W. Shaw, C. Pierre, Normal modes for non-linear vibratory systems, Journal of Sound and Vibration 164 (1) (1993) 85-124.

[11] S.W. Shaw, C. Pierre, Normal modes of vibration for non-linear continuous systems, Journal of Sound and Vibration 169 (3) (1994) 319-347.

[12] R.M. Rosenberg, On non-linear vibrations of systems with many degrees of freedom, Advances in Applied Mechanics 9 (1966) 155-242.

[13] A.F. Vakakis, L.I. Mikhlin, Y.V. Philipchuck, A.A. Zevin, Normal Modes and Localization in Nonlinear Systems, Wiley, New York, 1996.

[14] L. Jezequel, C.H. Lamarque, Analysis of non-linear dynamical systems by the normal form theory, Journal of Sound and Vibration 149 (3) (1991) 429-459.

[15] L. Jezequel, C.H. Lamarque, Nonlinear modal synthesis based on normal form theory, Proceedings of the Ninth International Modal Analysis Conference, Florence, Italy, 1991, pp. 101-108.

[16] A.H. Nayfeh, S.A. Nayfeh, On nonlinear modes of continuous systems, Transactions of American Society of Mechanical Engineers, Journal of Vibration and Acoustics 116 (1994) 129-136.

[17] R.H. Rand, A direct method for non-linear normal modes, International Journal of Non-linear Mechanics 9 (1974) 363-368.

[18] A.F. Vakakis, Non-linear normal modes (NNMs) and their applications in vibration theory: an overview, Mechanical Systems and Signal Processing 11 (1) (1997) 3-22.

[19] E. Peshek, C. Pierre, S.W. Shaw, A new Galerkin-based approach for accurate non-linear normal modes through invariant manifolds, Journal of Sound and Vibration 249 (5) (2002) 971-993.

[20] J. Guckenheimer, P.J. Holmes, Non-linear Oscillations, Dynamical Systems and Bifurcations of Vector Fields, Springer, New York, 1986.

[21] V.I. Arnold, Chapitres Supplémentaires de la Théorie des Équations Différentielles Ordinaires, Mir/Librairie du Globe, Moscow/Paris, 1980.

[22] H. Poincaré, Les Méthodes Nouvelles de la Mécanique Céleste, Gauthiers-Villars, Paris, 1892.

[23] A.D. Brjuno, Analytical form of differential equations, Transactions of Moscow Mathematical Society 25 (1971) $131-288$. 
[24] A.H. Nayfeh, Method of Normal Forms, Wiley, New York, 1993.

[25] F. Pellicano, F. Mastroddi, Applicability conditions of a non-linear superposition technique, Journal of Sound and Vibration 200 (1) (1997) 3-14.

[26] L. Morino, F. Mastroddi, M. Cutroni, Lie Transformation method for dynamical systems having chaotic behaviour, Nonlinear Dynamics 7 (1995) 403-428.

[27] A.H. Nayfeh, C. Chin, S.A. Nayfeh, On nonlinear modes of systems with internal resonance, Transactions of American Society of Mechanical Engineers, Journal of Vibration and Acoustics 118 (1996) 340-345.

[28] E. Peshek, C. Pierre, A global methodology for the modal reduction of large non-linear systems containing quadratic and cubic non-linearities, Proceedings of DETC'97, American Society of Mechanical Engineers Design Engineering Technology Conference, Sacramento, CA, 1997.

[29] T.D. Burton, M.N. Hamdan, On the calculation of non-linear normal modes in continuous systems, Journal of Sound and Vibration 197 (1) (1996) 117-130.

[30] M.I. Qaisi, Non-linear normal modes of a continuous system, Journal of Sound and Vibration 209 (4) (1998) 561-569.

[31] F. Pellicano, F. Mastroddi, Nonlinear dynamics of a beam on elastic foundation, Nonlinear Dynamics 14 (1997) 335-355.

[32] C. Touzé, A normal form approach for non-linear normal modes, Internal Publication of the LMA, Vol. 156, Marseille.

[33] C. Touzé, O. Thomas, Determination of non-linear normal modes for conservative systems, Proceedings of the Ninth ICSV, Orlando, Paper N. 70, 2002.

[34] C. Touzé, O. Thomas, Modes normaux non-linéaires de systèmes continus, Proceedings of Congrès Français de Mécanique, Nice, 2003.

[35] P. Manneville, L.S. Tuckerman, Phenomenological modeling of the first bifurcations of a spherical Couette flow, Journal de Physique 48 (1987) 1461-1469.

[36] N. Boivin, C. Pierre, S.W. Shaw, Non-linear modal analysis of structural systems featuring internal resonances, Journal of Sound and Vibration 182 (2) (1995) 336-341.

[37] A.G. Haddow, A.D.S. Barr, D.T. Mook, Theoretical and experimental study of modal interaction in a two-degreeof-freedom structure, Journal of Sound and Vibration 97 (3) (1984) 451-473.

[38] S. Sridhar, D.T. Mook, A.H. Nayfeh, Nonlinear resonances in the forced responses of plates, Part I: symmetric responses of circular plates, Journal of Sound and Vibration 41 (3) (1975) 359-373.

[39] C. Touzé, P. Lanchantin, O. Thomas, A. Chaigne, Transferts d'énergie par couplage modal: étude d'un cas particulier, Proceedings of Sixième Congrès Français d'Acoustique, Lille, 2002. 